DRAFT VERSION JANUARY 10, 2017

Preprint typeset using $\mathrm{LAT}_{\mathrm{E}} \mathrm{X}$ style AASTeX6 v. 1.0

\title{
TYPE Ib AND IIb SUPERNOVA PROGENITORS IN INTERACTING BINARY SYSTEMS
}

\author{
Sung-CHul YoON ${ }^{1}$ \\ Department of Physics and Astronomy, Seoul National University, Gwanak-ro 1, Gwanak-gu, Seoul, 08826, Korea \\ LuC DESSART \\ Unidad Mixta Internacional Franco-Chilena de Astronomía (CNRS UMI 3386), Departamento de Astronomía, Universidad de Chile, \\ Camino El Observatorio 1515, Las Condes, Santiago, Chile \\ Alejandro Clocchiatti \\ Instituto de Astrofísica, Pontificia Universidad Católica de Chile and Millennium Institute of Astrophysics, Chile

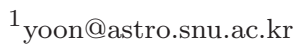

\begin{abstract}
We explore properties of Type Ib and IIb SN progenitors that are produced by stable mass transfer in binary systems, using a new grid of stellar evolution models from an initial primary mass in the range of $10-18 \mathrm{M}_{\odot}$ at solar and Large Magellanic Cloud metallicities. We find that blue and yellow supergiant SN IIb progenitors (e.g., of SN 2008ax, SN 2011dh, SN 2016gkg) have a hydrogen envelope mass less than about $0.15 \mathrm{M}_{\odot}$, mostly resulting from early Case B mass transfer with relatively low initial masses and/or low metallicity. Red supergiant (RSG) SN IIb progenitors (e.g., of SN 1993J, SN 2013df) are produced via late Case B mass transfer and have a more massive hydrogen envelope $\left(M_{\mathrm{H} \text {,env }}>0.15 \mathrm{M}_{\odot}\right)$. SN Ib progenitors are predominantly produced by early Case $\mathrm{B}$ mass transfer. Our models predict that SN IIb progenitors are systematically more luminous in the optical $\left(-8.0 \lesssim M_{\mathrm{V}} \lesssim-5.0\right)$ than the majority of SN Ib progenitors $\left(M_{\mathrm{V}} \gtrsim-5.0\right)$ for our considered initial mass range. However, the optically bright progenitor of SN Ib iPTF13bvn (i.e., $M_{\mathrm{V}} \simeq-6.5$ ) can be well explained by a relatively low-mass progenitor with a final mass of $\sim 3.0 \mathrm{M}_{\odot}$. The event rate of blue and yellow SN IIb progenitors would increase as metallicity decreases, while the event rate of SN Ib progenitors would decrease instead. By contrast, the population of RSG SN IIb progenitors would not be significantly affected by metallicity.
\end{abstract}

Keywords: stars: evolution - binaries: general - supernovae: general

\section{INTRODUCTION}

Type IIb supernovae ( $\mathrm{SNe}$ ) belong to the class of hydrogen-poor core-collapse SNe. They appear as a Type II SN initially but gradually turn into a Type Ib SN days to weeks after the explosion. This property can be best explained by a progenitor star having a small amount of hydrogen $\left(M_{\mathrm{H} \text {,env }} \approx 0.01-1.0 \mathrm{M}_{\odot}\right)$ in the envelope.

The inferred pre-explosion masses of Type IIb SN (hereafter, SN IIb) progenitors are typically about 3 - $6 \mathrm{M}_{\odot}$ (e.g., SN 1993J, Woosley et al. 1994; Shigeyama et al. 1994, Blinnikov et al. 1998; SN 2008ax, Taubenberger et al. 2011; Folatelli et al. 2015; SN 2011dh, Bersten et al. 2012; Ergon et al. 2015; SN 2011fu, Kumar et al. 2013; SN 2011ei, Milisavljevic et al. 2013; SN 2011hs, Bufano et al. 2014; SN 2013df, Szalai et al. 2016). This implies that their immediate progenitors are not massive Wolf-Rayet (WR) stars of WNh type, which are more massive than 8 -
$10 M_{\odot}$, and that the initial mass of a SN IIb progenitor is in the range of $10-18 \mathrm{M}_{\odot}$. It is therefore most likely that SN IIb progenitors have their hydrogen envelope mainly removed by Roche-lobe overflow rather than by stellar wind mass loss (Ensman \& Woosley 1988; Podsiadlowski et al. 1993; Stancliffe \& Eldridge 2009; Claeys et al. 2011; Dessart et al. 2011; Bersten et al. 2012; Benvenuto et al. 2013; Dessart et al. 2015; Folatelli et al. 2015; Dessart et al. 2016).

The progenitors of a few SNe IIb have been directly identified in pre-SN images as supergiants with a radius $\gtrsim 200 \mathrm{R}_{\odot}$ (i.e., SN 1993J, SN 2011dh, and SN 2013df; Aldering et al. 1994; Maund et al. 2004, 2011; Van Dyk et al. 2011, 2014). Comparison with stellar evolution and supernova models implies that these progenitors had an inflated hydrogen envelope of a small mass. The luminosities of these progenitors are in the range of $\log L / \mathrm{L}_{\odot}=4.92-5.12$. The corresponding intial mass 
is in the range of $11-17 \mathrm{M}_{\odot}$, which is consistent with the inferred pre-explosion masses of $3-6 \mathrm{M}_{\odot}$. The inflated hydrogen envelope of supergiant progenitors significantly influences the early-time light curves of $\mathrm{SNe} \mathrm{IIb}$ during the shock-cooling phase. In particular, several studies conclude that the sustained high luminosity at early times observed in some $\mathrm{SNe}$ IIb is related to this inflated hydrogen envelope (e.g., SN 1993J, SN 2011hs, SN 2011fu \& SN 2013df; Bersten et al. 2012; Nakar \& Piro 2014; Morozova et al. 2015).

However, observations indicate that not all SNe IIb have a supergiant progenitor. For example, very weak or no sign of sustained brightness at early times before the rise to the ${ }^{56} \mathrm{Ni}$-powered peak in the light curve is found for SN 1996cb and 2008ax (Qiu et al. 1999; Pastorello et al. 2008), which implies their progenitors were fairly compact. The analysis of SN 2008ax by Folatelli et al. (2015) indicates that its progenitor had a radius of $R \lesssim 50 \mathrm{R}_{\odot}$ and a pre-explosion mass of about $4-5 \mathrm{M}_{\odot}$. Evolutionary models with initial masses of $13-18 \mathrm{M}_{\odot}$ also indicate that solutions for compact SN IIb progenitors $\left(R \lesssim 50 \mathrm{M}_{\odot}\right)$ can be obtained with binary systems of relatively short initial periods $\left(P_{\text {init }} \lesssim \sim\right.$ 10 d; Yoon et al. 2010; Folatelli et al. 2015), while redsupergiant progenitors can be produced with much longer initial periods $\left(P_{\text {init }}=\sim 1000 \mathrm{~d}\right.$; Podsiadlowski et al. 1993; Stancliffe \& Eldridge 2009; Claeys et al. 2011). A yellow supergiant progenitor could be obtained with an initial period of $\sim 100$ d (Stancliffe \& Eldridge 2009; Bersten et al. 2012; Benvenuto et al. 2013). The observational diversity of the signatures of interaction with circumstellar matter for some $\mathrm{SNe}$ IIb may also reflect different histories of mass loss due to different progenitor properties (e.g., Chevalier \& Soderberg 2010; Ben-Ami et al. 2015; Maeda et al. 2015; Kramble et al. 2016).

The stellar evolution studies quoted above mostly focused on explaining specific cases for SN IIb progenitors. The purpose of this study is to have a more comprehensive view on the properties of SN IIb progenitors and to discuss the observational diversity of SNe IIb. For this purpose, we present a new grid of binary star evolution covering a range of initial primary-star mass (10 $\left.-18 \mathrm{M}_{\odot}\right)$ and a range of initial period $(\sim 10-3000 \mathrm{~d})$ for two different metallicities (solar and Large Magellanic Cloud values). Although our discussion is focused on SN IIb progenitors, SNe IIb and Ib form a continuous sequence of the increasing loss of the hydrogen-rich envelope and therefore represent a unique family (distinct from SNe Ic). In this context, our model grid includes $\mathrm{SNe} \mathrm{Ib}$ progenitor models for which no hydrogen is left in the envelope. We compare their properties at the final stage with those of SN IIb progenitors. This complements our previous work on the properties of $\mathrm{SN} \mathrm{Ib/c}$ progenitors (Yoon et al. 2010, 2012; Kim et al. 2015).

This paper is organized as follows. We present the numerical method and physical assumptions adopted for our evolutionary models in Section 2. The main results are presented in Section 3, The properties of SN IIb and SN Ib progenitors at the final stage are discussed in Sections 4 and 5 , respectively. The effect of metallicity on progenitor properties is addressed in Section 6. We conclude our study in Section 7.

\section{NUMERICAL METHOD AND PHYSICAL ASSUMPTIONS}

The model grid presented in this study has been calculated with the MESA code (Paxton et al. 2011, 2013, 2015). We adopt the Schwarzschild criterion for convection. We consider convective overshoot using a step function over a layer of thickness $l_{\mathrm{OV}}=0.3 H_{P}$ above the hydrogen burning convective core, where $H_{P}$ is the pressure scale height at the outer boundary of the core. We use the Dutch scheme in MESA for both hot and cool wind mass loss rates, with the Dutch scaling factor of 1.0 .

The energy transport efficiency by convection is usually parameterized by the mixing length $l_{\mathrm{m}}$ in units of $H_{P}$. We adopt the default value of mesa $\left(l_{\mathrm{m}}=1.5 H_{P}\right)$. We do not employ the artificial boost of convective energy transport using an excessive temperature gradient in the convective envelope that was introduced by Paxton et al. (2013).

We employ the optically thick mass transfer prescription by Kolb \& Ritter (1990) for calculating the mass transfer rate. This is well suited for the case of mass transfer from a supergiant star whose pressure scale height in the outer layers may become comparable to the size of the star (Pastetter \& Ritter 1989). The standard Ritter scheme (Ritter 1988) can be applied only if the pressure scale height at the stellar surface is much smaller than the stellar radius $\left(H_{P} \ll R\right)$.

We do not consider rotation in this study, but assume non-conservative mass transfer as predicted by previous binary models including the effects of rotation (Petrovic et al. 2005; Yoon et al. 2010; Langer 2012). In these studies, the mass accretor is quickly spun up to critical rotation during a mass transfer phase, and the stellar wind mass loss rate from the accretor dramatically increases accordingly, which effectively results in highly non-conservative mass transfer. In our calculation, therefore, we assume the ratio of the accreted mass to the transferred mass (i.e., the mass accretion efficiency $\beta)$ is 0.2 . In this picture, the non-accreted matter is blown away from the binary system as a fast wind from the mass accretor, and the corresponding angular momentum loss from the orbit is calculated accordingly.

We considered four different primary star masses and 


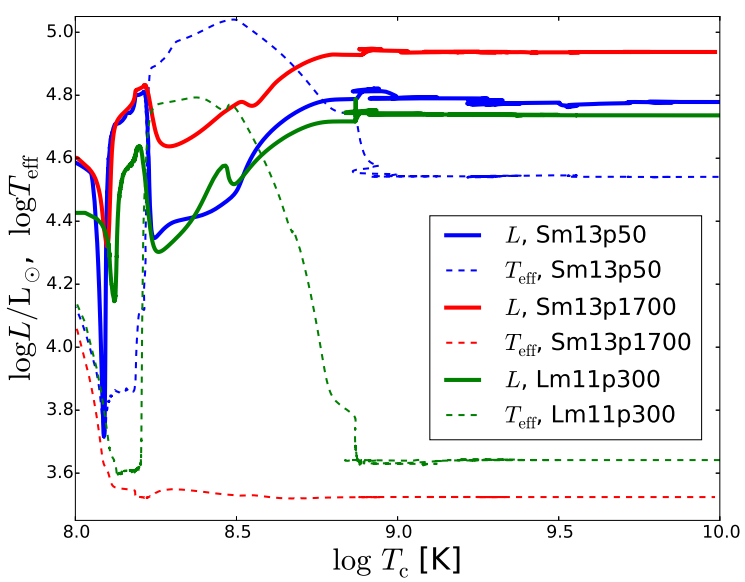

Figure 1. Evolution of the luminosity (solid line) and surface temperature (dashed line) of the primary star in Sm13p50 (blue), Sm13p1700 (red) and Lm11p300 (green), as a function of temperature at the center. The luminosity and surface temperature remain almost constant after $T_{\mathrm{c}}$ increases beyond $10^{9} \mathrm{~K}$.

two different metallicities: $M_{1}=11,13,16$, and $18 \mathrm{M}_{\odot}$, and $Z=0.02$ and 0.007 , which roughly correspond to solar and Large Magellanic Cloud (LMC) metallicities respectively. The mass ratio is fixed to $q=0.9$ for all models in our grid. To make a robust prediction on the event rates of $\mathrm{SNe} \mathrm{Ib}$ and IIb from binary systems, we would need to consider a wider range of mass ratio (Claeys et al. 2011), but it is beyond the scope of this paper.

The properties of the primary star at the end of the calculation for each sequence are summarized in Tables 7, 7, and 7. LMC and solar metallicity models have reference names starting with L and S, respectively (Lm11p100, Sm13p500, etc.). Names starting with $\mathrm{T}$ refer to test models with a reduced mass loss rate at solar metallicity (e.g., Tm11p20; see Section 3.3). Each name also contains the information of the initial mass of the primary star and the initial period. For example, Lm11p100 refers to the sequence with $M_{1 \text {,init }}=11 \mathrm{M}_{\odot}$ and $P_{\text {init }}=100 \mathrm{~d}$ at LMC metallicity.

The model evolution is halted when the central temperature $T_{\mathrm{c}}$ reaches $10^{9} \mathrm{~K}$ in most cases. The evolution of Lm11p300, Sm13p50 and Sm13p1700 is calculated to the pre-SN stage to check if there would be any significant change in the surface properties (i.e., $L$ and $T_{\text {eff }}$ ) from this point. As shown in Fig. 1, the envelope structure remains almost unchanged, because of very short evolutionary time from $T_{\mathrm{c}}=10^{9} \mathrm{~K}$ until core-collapse ( $10 \mathrm{yr}$ ). In the sequences Sm10p50 and Sm10p200, the carbon-oxygen core becomes electron-degenerate as its mass approaches $1.4 \mathrm{M}_{\odot}$. Off-center oxygen burning starts in these models before reaching $T_{\mathrm{c}}=10^{9} \mathrm{~K}$, and we stopped the calculation at this point because the time step becomes extremely small. It is likely that these models result in SN Ib progenitors, but we leave their final fate open in the present study.

\section{RESULTS}

Within the parameter space we considered in the present study, the primary star undergoes Case B mass transfer: Roche-lobe overflow after core hydrogen exhaustion and before core helium exhaustion. For our discussion, we further distinguish Case B as follows:

- Case EB (early Case B): mass transfer while the primary star crosses the Hertzsprung gap on the HR diagram.

- Case LB (late Case B): mass transfer after the hydrogen envelope of the primary star becomes fully convective.

After Case EB/LB mass transfer, a second mass transfer from the primary star may occur after core helium exhaustion. This case is referred to as Case EBB/LBB.

We can roughly predict the resultant SN type from our models based on their structures. A SN IIb progenitor must contain some hydrogen in the envelope but the hydrogen envelope mass $\left(M_{\mathrm{H} \text {,env }}\right)$ should not exceed a certain limit as otherwise the resultant supernova would appear as a SN IIL or IIP. This upper limit of $M_{\mathrm{H}, \text { env }}$ would depend on the supernova parameters including supernova energy, total ejecta mass, and chemical composition. In the present study, we assume the condition of $M_{\mathrm{H}, \mathrm{env}} \leq 1.0 \mathrm{M}_{\odot}$ for $\mathrm{SN}$ IIb progenitors.

In addition, we categorize our SN IIb progenitor models into three groups according to their surface temperatures at the final stage as the following:

- SN IIb-B: blue progenitors $\left(\log T_{\text {eff }} / \mathrm{K}>3.875\right.$; e.g. SN 2008ax and SN 2016gkg) ${ }^{1}$

- SN IIb-Y: yellow supergiant (YSG) progenitors (3.681 $\leq \log T_{\text {eff }} / \mathrm{K} \leq 3.875$; e.g., SN 2011dh)

- SN IIb-R: red supergiant (RSG) progenitors ( $\log T_{\text {eff }} / \mathrm{K}<3.681$; e.g., SN 1993J and SN 2013df)

Our temperature criterion for YSGs is adopted from Drout et al. (2009). Note that in the literature, there is ambiguity about the definition of YSGs: some authors consider progenitors of SN 1993J and 2013df as YSGs (e.g., Bersten et al. 2012), which are RSGs according to our definition. In the case where no hydrogen

${ }^{1}$ Some of SN IIb-B models in our grid are too compact $(R<\sim$ $10 \mathrm{R}_{\odot}$ ) to be called a giant star, and therefore we do not refer our blue progenitor models to blue supergiant progenitors. 

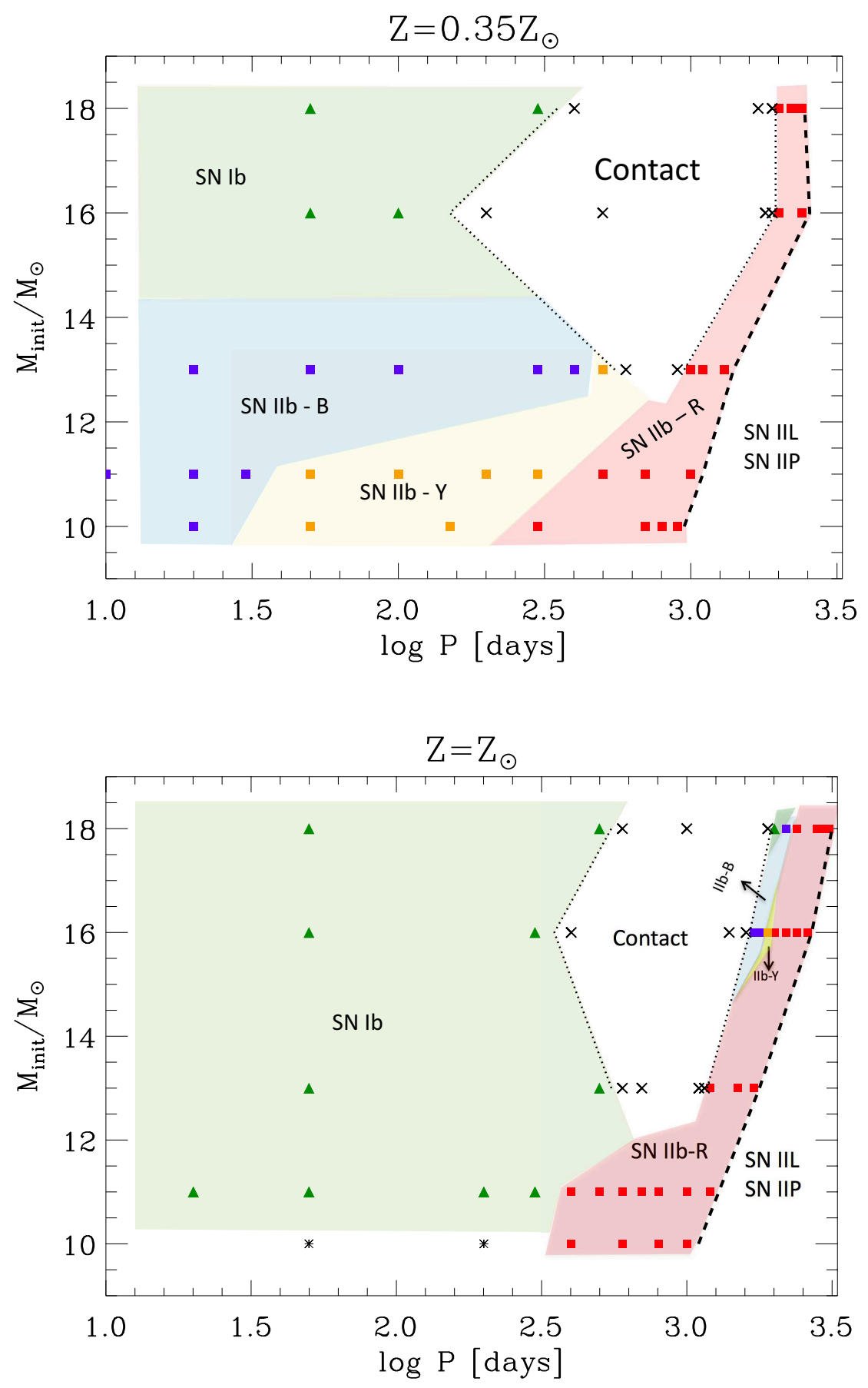

Figure 2. Grid points of the calculated binary sequences for LMC (upper panel) and solar (lower panel) metallicities on the plane spanned by the initial orbital period and the initial mass of the primary star. The predicted final fates of the primary stars are marked by different symbols. Triangle and square symbols denote SN Ib and SN IIb, respectively. Blue, orange and red colors for the square symbol indicate SN IIb progenitors of blue (SN IIb-B), yellow (SN IIb-Y) and red (SN IIb-R) colors, respectively (see the text). Sequences where the binary system becomes a contact binary are marked by a black cross. The black star symbols correspond to the models whose evolution was stopped before reaching $T_{\mathrm{c}}=10^{9} \mathrm{~K}$. 
is left, we assume that a $\mathrm{SN} \mathrm{Ib}$ would occur. The remaining helium mass in this case is higher than about $1.5 \mathrm{M}_{\odot}$, for which helium cannot be easily hidden in supernova spectra for the final mass range of our $\mathrm{SN} \mathrm{Ib}$ models $\left(3.0 \mathrm{M}_{\odot} \lesssim M \lesssim 6.6 \mathrm{M}_{\odot}\right.$; Hachinger et al. 2012; Dessart et al. 2015).

We summarize the result of our calculations in Fig. 2, where the predicted final fate of the primary star is indicated in the plane spanned by the initial period and initial mass. As shown in the figure, SN IIb progenitors are expected for a larger parameter space at LMC metallicity than at solar metallicity. The figure also shows the dependence of the progenitor type on the initial orbital period $\left(P_{\text {init }}\right)$ and the initial mass of the primary star $\left(M_{\text {init }}\right)$, as explained below in greater detail.

\subsection{LMC metallicity models}

It is well known that an initially tighter orbit leads to a deeper stripping of the hydrogen envelope via mass transfer. This effect of the initial separation can be observed in Fig. 3, as an example with $M_{\text {init }}=11 \mathrm{M}_{\odot}$.

With relatively short initial orbits $\left(P_{\text {init }}=10 \mathrm{~d}\right.$ and $100 \mathrm{~d}$ ), Case EB mass transfer occurs. In this case, most of the hydrogen envelope remains radiative at the onset of mass transfer, and strong mass loss leads to thermal disequilibrium of the envelope. The luminosity of the primary star rapidly drops accordingly (e.g., see De Loore \& Doom 1992; Eggleton 2011) until the total mass decreases to about $7 \mathrm{M}_{\odot}$ and $8 \mathrm{M}_{\odot}$ for $P_{\text {init }}=10 \mathrm{~d}$ and $100 \mathrm{~d}$, respectively. The star gradually becomes brighter thereafter as the surface mass fraction of hydrogen decreases. Once the mass transfer stops, both the radius and the luminosity of the star decrease until thermal equilibrium is restored.

After core helium exhaustion, the envelope expands again until the end point. The surface temperature at the end point is $\log T_{\text {eff }} / \mathrm{K}=4.44$ and 3.79 for $P_{\text {init }}=10$ and $100 \mathrm{~d}$, respectively. As discussed in Section 4.3 below in more detail, the final surface temperature tends to be lower for a higher hydrogen envelope mass that remains until the end, which is in turn determined by the initial orbital separation.

In the sequences with $P_{\text {init }}=700 \mathrm{~d}$ and $1000 \mathrm{~d}$, mass transfer occurs when the hydrogen envelope of the primary star is fully convective (Case LB). The response to mass loss of a convective envelope is very different from that of a radiative envelope (De Loore \& Doom 1992; Eggleton 2011). As shown in Fig. 3, the radius initially increases once the primary star begins to transfer mass to its companion. In the sequence of $P_{\text {init }}=700 \mathrm{~d}$, after the mass transfer stops, the star undergoes a blueward evolution on the HR diagram. The star turns to the red again and becomes a RSG at the final stage. On the other hand, the HR diagram of the sequence with
$P_{\text {init }}=1000 \mathrm{~d}$ does not show the blue loop. This is because the hydrogen envelope mass remains sufficiently large to preserve the RSG structure.

In Fig. 4, the evolution of the mass transfer rate from the $11 \mathrm{M}_{\odot}$ and $18 \mathrm{M}_{\odot}$ primary stars is presented for three different initial periods. We note that Case EB mass transfer $\left(P_{\text {init }}=100 \mathrm{~d}\right.$ for $11 \mathrm{M}_{\odot}$ and $P_{\text {init }}=300 \mathrm{~d}$ for $18 \mathrm{M}_{\odot}$ ) lasts longer than Case LB mass transfer, which explains the deeper stripping of the hydrogen envelope with Case EB. With Case LB mass transfer, the duration and mass transfer rate become smaller with a longer initial period. There are two mass transfer phases for the $11 \mathrm{M}_{\odot}$ model. The first one is responsible for most of the stripping of the hydrogen envelope. The second one is a brief phase with a lower mass transfer rate, which occurs after core helium exhaustion as the primary star tends to expand during the final evolutionary stages.

This second mass transfer is less favored in more massive systems (Fig. 4; see also Tables 7, 7, and 7). This is because of the following two factors. 1) The Case EBB mass transfer (i.e., the second mass transfer after the Case EB mass transfer) is commonly observed for relatively low-mass stars (e.g., Yoon et al. 2010). The envelope expansion after core helium exhaustion is more prominent with a more compact stellar core. On the other hand, higher mass stars $\left(M_{\text {init }} \gtrsim 13 \mathrm{M}_{\odot}\right)$ tend to remain more compact provided $M_{\mathrm{H} \text {,env }}<0.15 \mathrm{M}_{\odot}$ after the Case EB mass transfer. 2) The Case LBB mass transfer (i.e., the second mass transfer after the Case LB mass transfer) is also driven by the expansion of the remaining hydrogen envelope. For more massive systems, however, stronger stellar winds tend to make the orbit wider, preventing the primary star from undergoing further mass transfer.

Although mass transfer is the main determining factor for the final envelope structure of SN progenitors in binary systems, the effect of wind mass loss can also play an important role. As discussed by Yoon et al. (2010), mass transfer cannot completely remove hydrogen from the primary star even with Case EB. Whether the progenitor can retain some hydrogen until core collapse depends on the subsequent history of mass loss by stellar winds. Stellar winds are stronger for higher masses, and no hydrogen is left in Case EB systems with $M_{\text {init }} \geq 16 \mathrm{M}_{\odot}$ at $Z=Z_{\mathrm{LMC}}$ (Fig. 2; Table 7).

With our assumption of $\beta=0.2$, the Case EB and Case LB regimes are separated by the contact regime for the sequences with $M \geq 13 \mathrm{M}_{\odot}$ (Fig. 2). With a larger value of $\beta$, the regime for contact would be enlarged.

In terms of the final fate (Fig. 2), solutions for SN IIb-B and SN IIb-Y progenitors are found only for $M \leq 13 \mathrm{M}_{\odot}$ in our considered parameter space. SN IIb-R solutions are found for all the considered mass range. The tendency that the surface temperature gradually decreases 

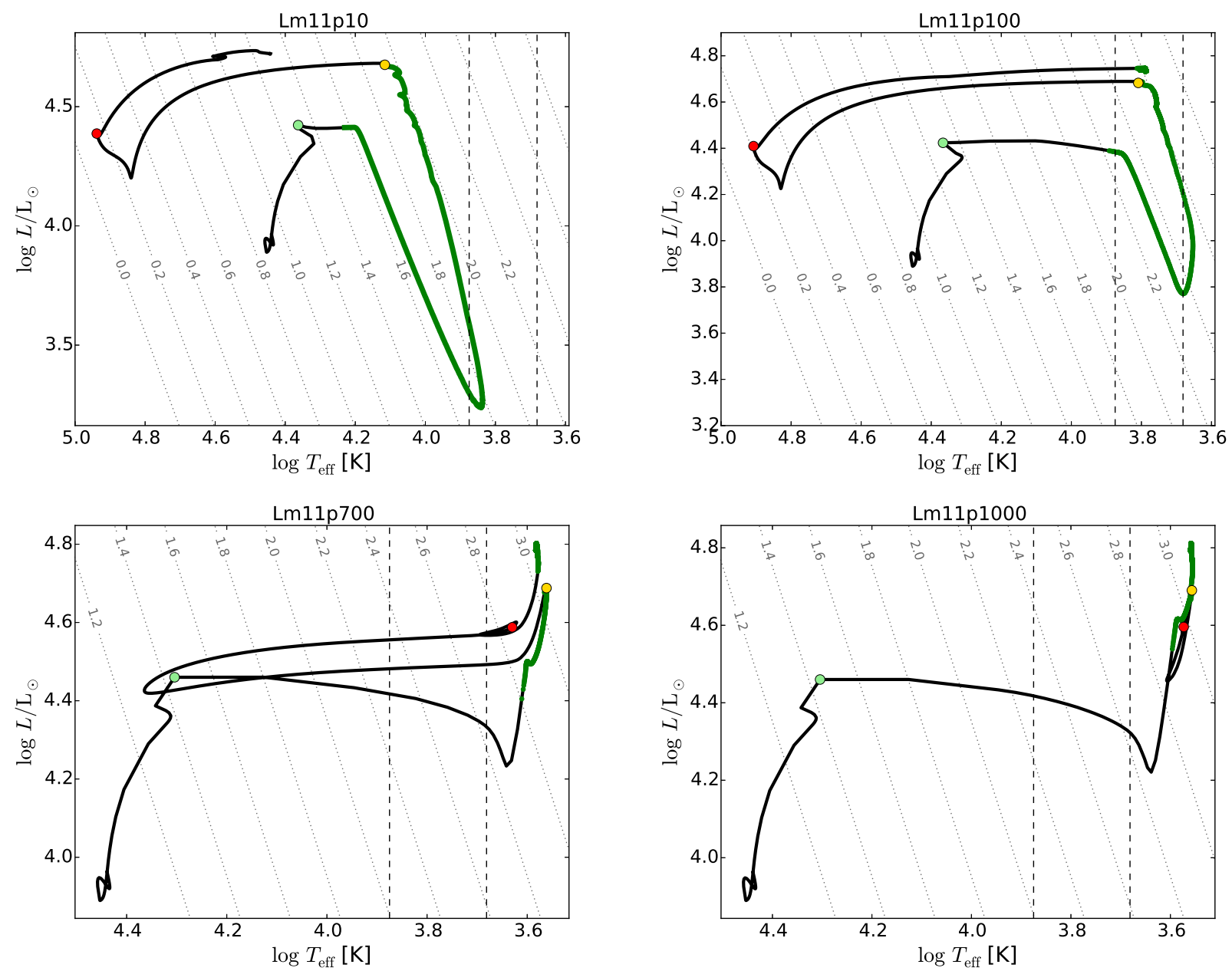

Figure 3. HR diagrams for systems with a primary star of $M_{\text {init }}=11 \mathrm{M}_{\odot}$ at LMC metallicity. The secondary mass is $9.9 \mathrm{M}_{\odot}$. The adopted mass accretion efficiency is 0.2 . The mass transfer phases are marked by green color on each evolutionary track. The initial periods of the systems are 10 (top left), 100 (top right), 700 (bottom left), and 1000 (bottom right) days, as indicated on top of each figure. The labels with dotted contour lines indicate log $R / \mathrm{R}_{\odot}$. The vertical dashed lines give the boundary values for yellow supergiants $\left(3.681 \leq \log T_{\text {eff }} / \mathrm{K} \leq 3.875\right)$. The light green, yellow and red circles denote core hydrogen exhaustion, onset of core helium burning and core helium exhaustion, respectively.

with increasing $P_{\text {init }}$ for a given initial mass can be explained by the fact that less hydrogen is removed in the case of a wider orbit.

\subsection{Solar metallicity models}

The mass transfer history of solar metallicity models is qualitatively similar to that of LMC metallicity models. A shorter initial orbit leads to more significant stripping of the hydrogen envelope, and vice versa. However, the role of stellar winds becomes more important, compared to the LMC case.

The most notable difference between solar and LMC metallicity models is observed in the outcome of Case EB mass transfer. As shown in Fig. 2, hydrogen is completely removed via stellar winds during the post Case EB mass transfer phase (see also Table 7). The solutions of SN IIb-B and SN IIb-Y that are found with LMC metallicity with $M \leq 13 \mathrm{M}_{\odot}$ are replaced by $\mathrm{SN}$ Ib solutions.

Strong winds also significantly affect the evolution with Case LB mass transfer. Narrow regimes for SN IIb-B solutions appear with $M=16 \mathrm{M}_{\odot}$ and $18 \mathrm{M}_{\odot}$, which are not found at LMC metallicity. This is because the primary star loses a significant amount of hydrogen during the blue loop phase where the WR mass loss rate of Nugis \& Lamers (2000) is applied (see Section 3.3 below) after Case LB mass transfer (Fig. 5). At $M_{\text {init }}=16 \mathrm{M}_{\odot}$, a SN IIb-Y solution is also found in-between SN IIb-B and SN IIb-R regimes (Figs. 2 and 5).

In general, RSG solutions are dominant for $\mathrm{SN}$ IIb progenitors at solar metallicity. However, many of the SN 

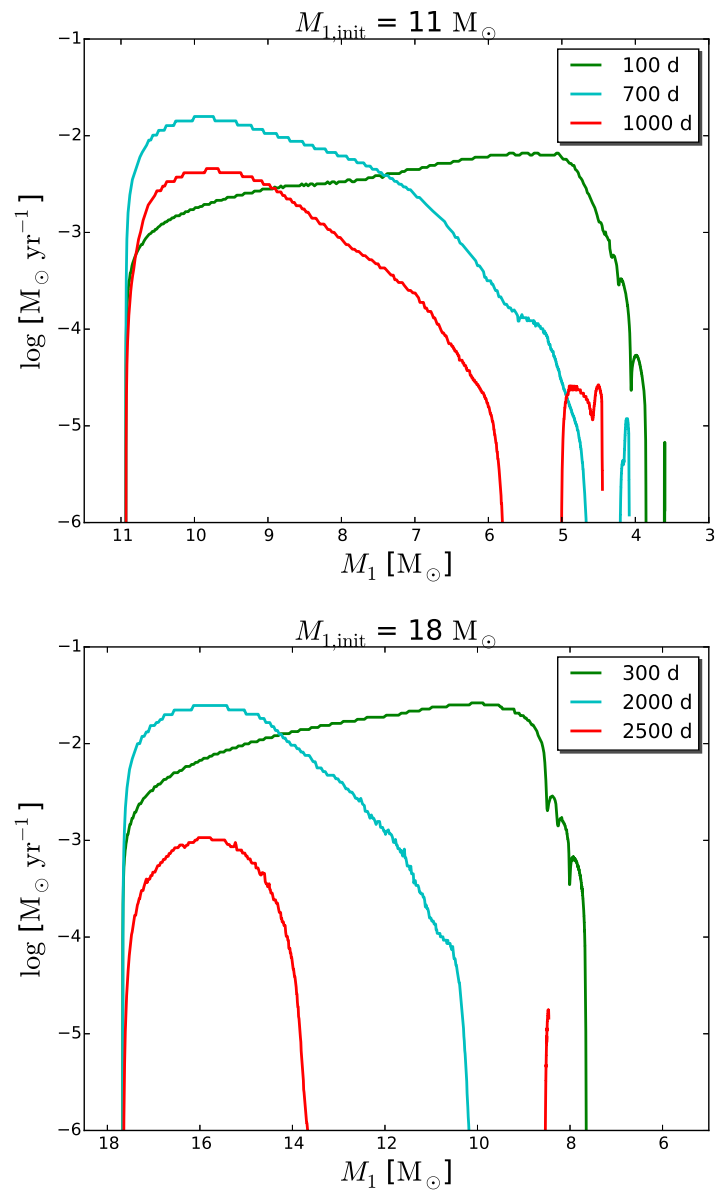

Figure 4. The mass transfer rates as a function of the total mass of the primary star, for systems with $M_{1 \text {,init }}=$ $11 \mathrm{M}_{\odot}$ (upper panel) and $M_{1, \text { init }}=18 \mathrm{M}_{\odot}$ (lower panel) at LMC metallicity. The initial periods are indicated by different colors.

IIb-R progenitor models have higher surface temperatures $\left(\log T_{\text {eff }} / \mathrm{K}>3.6\right)$ than those of ordinary RSGs observed in the local universe (see Table 7). This is consistent with the progenitor properties of SN 1993J and SN 2013df, for which $\log T_{\text {eff }} / \mathrm{K} \simeq 3.63$ was inferred observationally (Maund et al. 2004; Van Dyk et al. 2014).

\subsection{Effect of $W R$ winds}

As discussed above, the evolution of the primary star after the major mass transfer phase is critically affected by stellar wind mass loss. In particular, the primary star spends most of the remaining evolutionary time on the helium main sequence as a hydrogen-poor star after Case EB mass transfer. The WR mass loss rate by Nugis \& Lamers (2000) (hereafter, the NL rate) is applied for this case. However, the NL rate was inferred from Galactic WR stars, which are much more massive $\left(M \gtrsim 10 \mathrm{M}_{\odot}\right)$ and therefore much more luminous than our primary star models during the post mass transfer
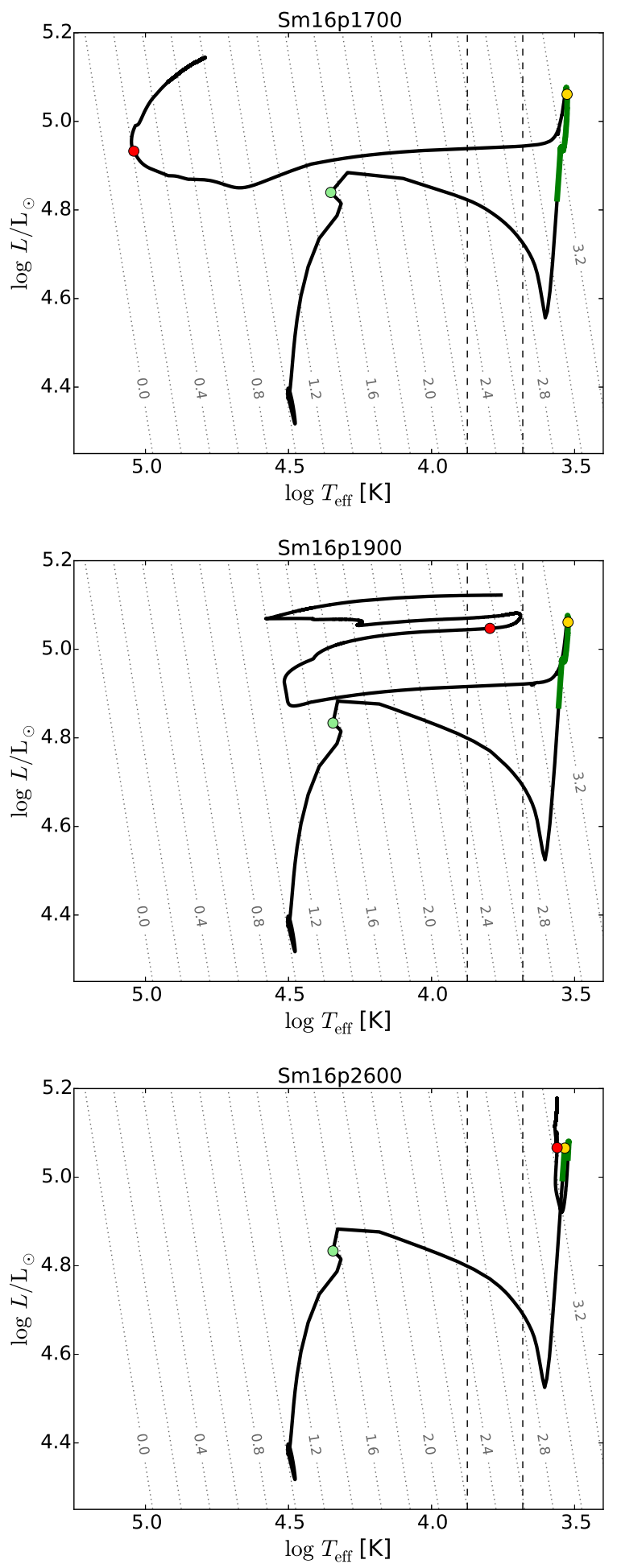

Figure 5. HR diagrams for systems with a primary star of $M_{\text {init }}=16 \mathrm{M}_{\odot}$ at solar metallicity. The secondary mass is $14.4 \mathrm{M}_{\odot}$. The initial periods of the systems are 1700 (top), 1900 (middle), and 2600 (bottom) days. The mass transfer phases are marked by green color on each evolutionary track. The labels with dotted contour lines indicate $\log R / \mathrm{R}_{\odot}$. The vertical dashed lines give the boundary values for yellow supergiants $\left(3.681 \leq \log T_{\text {eff }} / \mathrm{K} \leq 3.875\right)$. The light green, yellow and red circles denote core hydrogen exhaustion, onset of core helium burning and core helium exhaustion, respectively. 
phase $\left(3 \mathrm{M}_{\odot} \lesssim M \lesssim 7 \mathrm{M}_{\odot}\right)$. The validity of the NL rate for these relatively low-mass hydrogen-poor hot stars has hardly been investigated previously (see the discussion by Yoon 2015).

For their SN Ib progenitor models in binary systems, Yoon et al. (2010) have applied a lower mass loss rate than the $\mathrm{NL}$ rate, if $\log L / \mathrm{L}_{\odot}<4.5$ during the post mass transfer phase. As a result, a small amount of hydrogen is left in many of their SN Ib progenitor models, which can be considered as SN IIb-B progenitors according to our definition.

To investigate further the effect of wind mass loss, we made a model grid with a reduced mass loss rate using the scaling factor $\eta=0.48$ on the Dutch mass loss rate recipe in the MESA code, at solar metallicity (Table 7). The mass loss rate with this reduction factor becomes comparable to that of LMC metallicity with the fiducial value of $\eta=1.0$. As shown in Table 7 , this reduction of the mass loss rate gives a result similar to that of the LMC metallicity models. Solutions for SN IIb progenitors are found with the sequences with $M_{\text {init }}=11 \mathrm{M}_{\odot}$ and $13 \mathrm{M}_{\odot}$ for $P_{\text {init }} \leq 400 \mathrm{~d}$, for which SN Ib solutions are obtained with our fiducial mass loss rate. With $M_{\text {init }}=11 \mathrm{M}_{\odot}$, solutions for SN IIb-B, SN IIb-Y, and SN IIb-R can be found in the order of increasing initial periods. With $M_{\text {init }}=13 \mathrm{M}_{\odot}$, only $\mathrm{SN}$ IIb-B is predicted. This result is also consistent with those of Benvenuto et al. (2013) and Folatelli et al. (2015), who found a SN IIb-Y solution for SN 2011dh and a SN IIb-B solution for SN 2008ax, respectively, at solar metallicity. Apparently, these authors did not apply WR mass loss in their binary models.

This consideration should be taken as a caveat when we discuss the metallicity dependence of the predicted SN type (see Section 6)

\section{PROPERTIES OF SN IIb PROGENITORS}

\subsection{Final mass - luminosity relation}

A few SN IIb progenitors have been directly identified in pre-SN images, including those of SN 1993J, SN 2008ax, SN 2011dh, SN 2013bf and SN 2016gkg (Aldering et al. 1994; Maund et al. 2004, 2011; Van Dyk et al. 2011, 2014; Kilpatrick et al. 2016; Tartaglia et al. 2016). Their properties are summarized in Table 4. In these studies, the progenitor masses at the pre-SN stage were inferred from their bolometric luminosities.

In Fig. 6, we present the final mass-luminosity relation of our SN IIb progenitor models. The luminosity at the pre-SN stage correlates with the initial and final masses. For a given final luminosity, however, the scatter of the final mass can be as large as $1.0 \mathrm{M}_{\odot}$. The bolometric luminosity at the pre-SN stage is mainly determined by

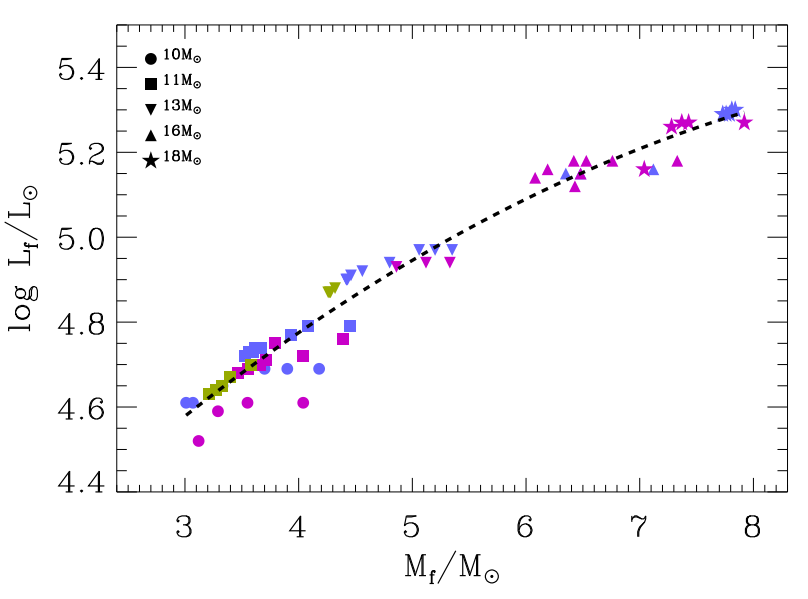

Figure 6. The final mass-luminosity relation of SN IIb progenitors. The initial primary mass is marked by different symbols: circle, square, inverted triangle, triange, and star denote $10,11,13,16$ and $18 \mathrm{M}_{\odot}$, respectively. Purple and blue indicate solar and LMC metallicities, respectively. Green gives the result with a reduced mass loss rate (i.e., $\eta=0.48$ ) at solar metallicity. The dashed line is the best fit of the data with a second order polynomial.

helium shell burning, which is in turn largely determined by the $\mathrm{CO}$ core mass. As shown in Tables 7, 7 and 7, different final $\mathrm{CO}$ core masses may result from different initial orbits for a given initial mass. This explains the scatter of the final masses for a given luminosity. Likewise, for a given initial mass, the final luminosity may vary by up to 0.2 dex in terms of $\log L / \mathrm{L}_{\odot}$.

The final mass-luminosity relation shown in Fig. 6 roughly gives $M_{f} \simeq 6.0,5.0$, and $4.9 \mathrm{M}_{\odot}$ for SN 1993J, SN 2011dh and SN 2013df, respectively, for which the progenitor luminosity is fairly well constrained. The corresponding initial masses are about $16 \mathrm{M}_{\odot}$ for SN 1993J and $13 \mathrm{M}_{\odot}$ for SN 2011dh and SN 2013df, in agreement with previous work.

\subsection{Final position on the HR diagram and optical brightness}

The positions on the HR diagram of our SN IIb progenitor models are shown in Fig. 7. Given the limited number of our models, this figure cannot properly reflect the real population of SN IIb progenitors. However, a few important features can be found here.

Note that RSG progenitors (SN IIb-R) like SN 1993J and SN 2013bf can exist for a wide range of luminosities (i.e, for a wide range of initial masses) regardless of metallicity, and can be relatively easily explained by Case LB mass transfer.

On the other hand, SN IIb-B and SN IIb-Y progenitors would be preferentially produced from relatively 

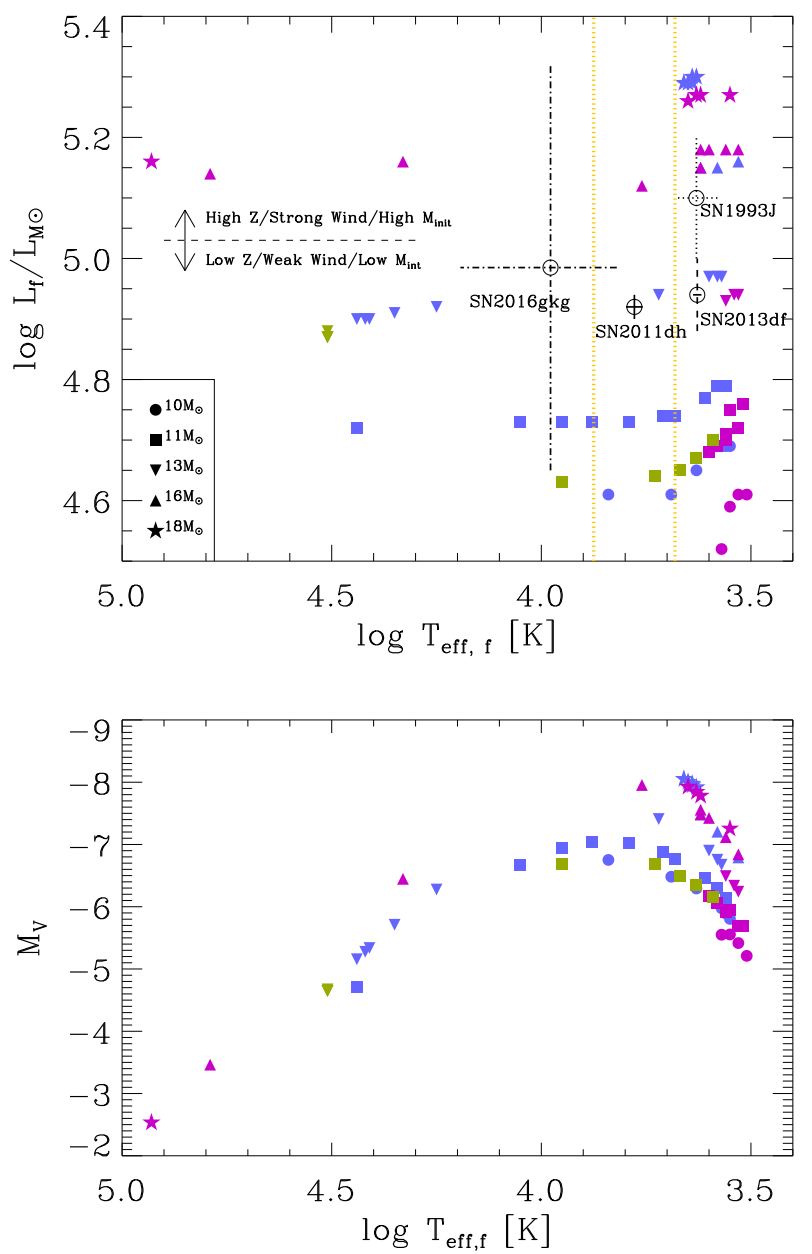

Figure 7. Upper panel: The final positions of our SN IIb models on the HR diagram. The initial primary mass is marked by different symbols: circle, square, inverted triangle, triangle, and star denote 10, 11, 13, 16 and $18 \mathrm{M}_{\odot}$, respectively. Purple and blue indicate solar and LMC metallicities, respectively. Green gives the result with a reduced mass loss rate (i.e., $\eta=0.48$ ) at solar metallicity. The orange dotted lines mark the boundaries for yellow supergiants: $3.681 \leq \log T_{\text {eff }} / \mathrm{K} \leq 3.875$. The black dashed line roughly gives the dividing line of SN IIb-B solutions for different metallicities, mass loss rates and/or initial masses (see the text for details). The open circles mark the positions of four observed SN IIb progenitors (SN 1993J, SN 2011dh, SN 2013df, and SN 2016gkg; see Table 4). Lower panel: Corresponding Vband magnitudes with black-body approximation.

low-mass progenitors, as also implied by Fig. 2. For $\log L_{f} / \mathrm{L}_{\odot}<5.0$, which corresponds to $M_{1, \text { init }} \lesssim 13 \mathrm{M}_{\odot}$ solutions for SN IIb-B and SN IIb-Y can be obtained only with LMC metallicity or with a reduced mass loss rate $(\eta=0.48)$ at solar metallicity. These solutions are obtained mostly with Case EB mass transfer. SN IIb-B and
IIb-Y solutions for $\log L_{f} / \mathrm{L}_{\odot}>5.0\left(M_{1 \text {,init }} \gtrsim 16 \mathrm{M}_{\odot}\right)$ result from strong mass loss after Case LB mass transfer, and are rarely achieved with $\eta=1.0$ at solar metallicity, as implied by Fig. 2.

The host galaxies of SN 2011dh and SN 2016gkg (M51 and NGC 613, respectively), for which the progenitors belong to SN IIb-B or SN IIb-Y type, are not particularly metal poor. This might imply a lower mass loss rate from the progenitors than the fiducial mass loss rate adopted in our study (cf. Benvenuto et al. 2013). However, the parameter space of our models is limited to stable mass transfer systems. In reality, multiple binary channels may exist (e.g., common envelope ejection) for $\mathrm{SN}$ IIb-B/IIb-Y progenitors, and this issue deserves a more detailed future study.

Fig. 7 also shows the V-band magnitudes of our SN IIb progenitor models, which were calculated with the blackbody approximation. In general, our models predict that SN IIb progenitors are much brighter $\left(M_{\mathrm{V}}<-6.0\right.$ for most cases) than hydrogen-free $\mathrm{SN} \mathrm{Ib/Ic} \mathrm{progenitors}$ $\left(M_{\mathrm{V}}>-4.5\right.$; Yoon et al. 2012; see Section 5 below) in the optical. Therefore, it is not surprising that SN IIb progenitors have be more commonly identified than SN $\mathrm{Ib} / \mathrm{Ic}$ progenitors so far (see Smartt 2015, for a recent review), despite the fact that the $\mathrm{SN} \mathrm{Ib}$ event rate is comparable to the SN IIb rate (e.g., Smith et al. 2011; Eldridge et al. 2013; Shivvers et al. 2016).

Note also that the optical brightness of SN IIb progenitors gradually decreases as the surface temperature increases for $\log T_{\text {eff }} / \mathrm{K} \gtrsim 3.9$. This is mainly because a larger bolometric correction is needed for a higher $T_{\text {eff }}$. In particular, SN IIb-B progenitors with $\log T_{\text {eff }} / \mathrm{K} \gtrsim 4.5$ have $M_{\mathrm{V}}>-5$ and would be relatively difficult to detect in pre-SN images, compared to SN IIb-Y and IIb-R progenitors.

\subsection{Final hydrogen mass - surface temperature relation}

One of the dominant factors that determine the final size and surface temperature of a SN IIb progenitor is the hydrogen envelope mass $\left(M_{\mathrm{H}, \text { env }}\right)$ at the pre$\mathrm{SN}$ stage. We find that $\mathrm{SN}$ IIb-R solutions are dominant if $M_{\mathrm{H} \text {,env }}>0.15 \mathrm{M}_{\odot}$, while $\mathrm{SN} \mathrm{IIb-Y}$ and $\mathrm{SN}$ IIb-B solutions are more common with a smaller hydrogen envelope mass (Fig. 8). This is in good agreement with the previous estimates for the SNe IIb in Table 4: $M_{\mathrm{H}, \text { env }}=0.2-0.4 \mathrm{M}_{\odot}$ for $\mathrm{SN} 1993 \mathrm{~J}$ and $\mathrm{SN} 2013 \mathrm{df}(\mathrm{RSG}$ progenitor), $M_{\mathrm{H} \text {,env }} \approx 0.1 \mathrm{M}_{\odot}$ for $\mathrm{SN} 2011 \mathrm{dh}$ (YSG progenitor) and $M_{\mathrm{H}, \mathrm{env}} \approx 0.06 \mathrm{M}_{\odot}$ for $\mathrm{SN} 2008 \mathrm{ax}(\mathrm{BSG}$ progenitor).

This result is also generally consistent with the finding of Meynet et al. (2015), who discussed single star SN progenitors at solar metallicity with enhanced RSG mass loss rates. A few notable differences are also found as the following: 


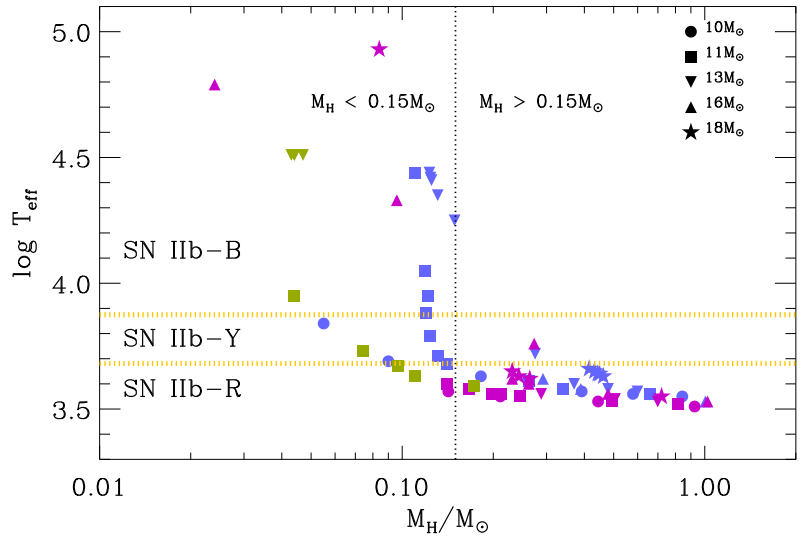

Figure 8. The final surface temperatures as a function of the hydrogen envelope mass $\left(M_{\mathrm{H}, \mathrm{env}}\right)$ of our $\mathrm{SN} \mathrm{IIb}$ progenitor models. The initial primary mass is marked by different symbols: circle, square, inverted triangle, triangle, and star denote 10, 11, 13, 16 and $18 \mathrm{M}_{\odot}$, respectively. Purple and blue indicate solar and LMC metallicities, respectively. Green gives the result with a reduced mass loss rate (i.e., $\eta=0.48$ ) at solar metallicity. The orange dotted lines mark the boundaries for yellow supergiants: $3.681 \leq \log T_{\text {eff }} / \mathrm{K} \leq 3.875$. The back dotted vertical line roughly defines the boundary between extended progenitors (SN IIb-R), and relatively compact progenitors (SN IIb-Y and SN IIb-B), in terms of the hydrogen envelope mass.

1. In Meynet et al., SN progenitors with $\log T_{\text {eff, }} / \mathrm{K}>$ 3.6 were found only with $M_{\mathrm{H} \text {,env }}<0.4 \mathrm{M}_{\odot}$, but in our grid, this upper limit extends to $M_{\mathrm{H}, \mathrm{env}}=$ $0.6 \mathrm{M}_{\odot}$.

2. Meynet et al. find SN progenitors with $\log T_{\text {eff, }} / \mathrm{K}<3.6$ only for $M_{\mathrm{H}, \text { env }}>0.9 \mathrm{M}_{\odot}$, but in our study, solutions with $\log T_{\text {eff,f }} / \mathrm{K}<3.6$ are obtained even for smaller hydrogen masses $\left(M_{\mathrm{H}, \mathrm{env}} \gtrsim\right.$ $\left.0.2 \mathrm{M}_{\odot}\right)$.

3. In Meynet et al., no models with $M_{\mathrm{H} \text {,env }}>$ $0.1 \mathrm{M}_{\odot}$ have a surface temperature higher than $\log T_{\text {eff }, \mathrm{f}} / \mathrm{K}=4.3$. By contrast, many of our LMC metallicity models have $\log T_{\text {eff, } \mathrm{f}} / \mathrm{K}>4.3$ with $M_{\mathrm{H}, \mathrm{env}}=0.1-0.14 \mathrm{M}_{\odot}$.

4. Our models include SN IIb-B progenitors with $\log T_{\text {eff,f }} / \mathrm{K} \geq 4.8$ at solar metallicity, which was not found in Meynet at al..

These differences can be explained by the fact that our models cover a wider parameter space than in Meynet et al., in terms of mass loss histories and metallicities.

4.4. Asymmetry of the progenitor envelope and winds

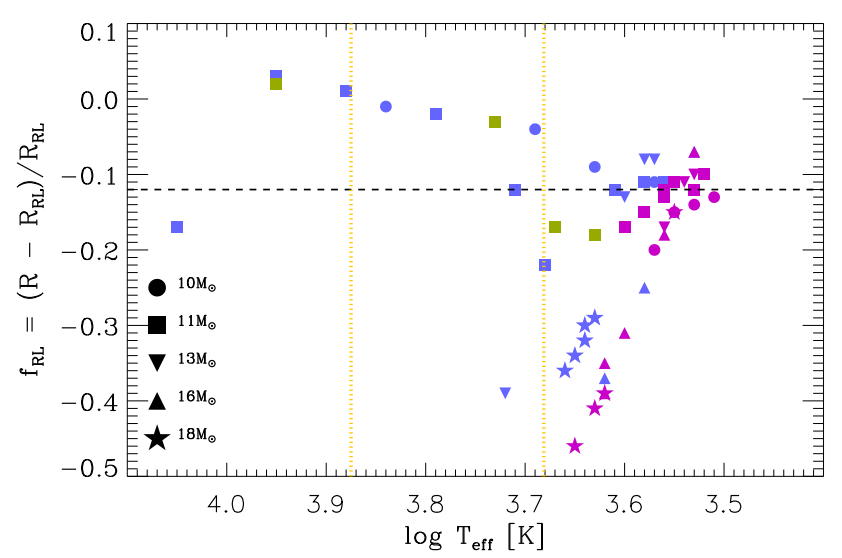

Figure 9. The Roch-lobe filling factor for the last computed models of primary stars, defined by $f_{\mathrm{RL}}=(R-$ $\left.R_{\mathrm{RL}}\right) / R_{\mathrm{RL}}$ where $R$ and $R_{\mathrm{RL}}$ denote the radius of the star and the Roche lobe radius, respectively. The orange dotted lines mark the boundaries for yellow supergiants: $3.681 \leq \log T_{\text {eff }} / \mathrm{K} \leq 3.875$.

Compared to single stars, the outermost layers of SN progenitors in binary systems can have an asymmetric structure at the pre-SN stage if they are filling or almost filling the Roche lobe. Mass transfer and interaction between stellar winds and transferred mass may also create complex, non-spherical circumstellar structures. These factors might be related to some polarization features observed at early times in SNe IIb when the photosphere is still located at the outermost ejecta layers (Höflich 1995; Tran et al. 1997; Maund et al. 2007; Chornock et al. 2011; Dessart \& Hillier 2011; Mauerhan et al. 2015; Stevance et al. 2016; cf. Reilly et al. 2016 for the SN Ib iPTF13bvn).

We present the Roche lobe filling factor $f_{\mathrm{RL}}$ for our SN IIb progenitor models in Fig. 9, which is defined as $f_{\mathrm{RL}}=\left(R-R_{\mathrm{RL}}\right) / R_{\mathrm{RL}}$, where $R_{\mathrm{RL}}$ is the Roche lobe radius. Note that mass transfer becomes significant $\left(\dot{M}_{\mathrm{tr}} \gtrsim 10^{-6} \mathrm{M}_{\odot} \mathrm{yr}^{-1}\right)$ if $f_{\mathrm{RL}} \gtrsim-0.12$, according to the adopted mass transfer scheme in our calculations (see Section 2). Many of our SN IIb progenitor models end their lives as a detached binary, but not a small fraction of the sequences have $f_{\mathrm{RL}}>-0.12$ at the final stage, in particular for SN IIb-Y progenitors with $M_{1, \text { init }} \leq 11 \mathrm{M}_{\odot}$. On the other hand, all SN IIb-B progenitors with $\log T_{\text {eff }} / \mathrm{K} \gtrsim 4.0$ are detached from the Roche lobe because they are compact. SN IIb-R progenitors tend to be more detached with a higher mass and no progenitors with $M_{1 \text {,init }}=18 \mathrm{M}_{\odot}$ fills the Roche lobe at the pre-SN stage. This is because stronger wind mass loss from a more massive progenitor tends to make the orbit wider than the corresponding case with a less massive progenitor. 
Whether or not the Roche lobe filling envelope at the pre-SN stage can explain some features of polarization during an early $\mathrm{SN}$ epoch is an important subject of future work. Previous spectropolarimetry studies on core collapse SNe imply that polarisation features are strongly connected to asymmetric explosion (e.g., Wang \& Wheeler 2008). Although signatures of asymmetric explosions are more prominent in late-time observations, its impact on shock heating of the envelope and/or asymmetric distribution of nickel may also play an important role in the early-time SN evolution (Mauerhan et al. 2015). Therefore, the distorted shape of the envelope would not be the only possible explanation for early-time polarisation.

The wind mass loss rates from SN IIb progenitors range from $\log \dot{M}_{\mathrm{w}}\left[\mathrm{M}_{\odot} \mathrm{yr}^{-1}\right] \simeq-6.5$ to $\log \dot{M}_{\mathrm{w}}\left[\mathrm{M}_{\odot} \mathrm{yr}^{-1}\right] \simeq$ -4.8 , and increase with final mass and radius (see Tables 7,7 and 7). These mass loss rates are largely consistent with the values inferred from observations of SN IIb interactions with circumstellar medium (e.g., Maeda et al. 2014, 2015).

The mass transfer rates from the Roche-lobe filling progenitors largely depend on $f_{\mathrm{RL}}$ at the final stage, rather than on the final mass (see Tables 7, 7 and 7). In our assumption of non-conservative mass transfer, the transferred material that is not accreted onto the secondary star is supposed to be blown away from the binary system as a fast wind from the secondary star. On the other hand, the winds from Roche-lobe filling progenitors would be much slower than the winds from secondary stars. Even if the primary star is not filling the Roche lobe, slow and fast winds are expected from a SN IIb-Y/IIb-R progenitor and its dwarf companion, respectively. The resultant wind-wind collisions would make a complicated, clumpy circumstellar structure. This possibility and its observational signatures deserve future numerical studies.

\section{PROPERTIES OF SN Ib PROGENITORS}

Many of our considered systems produce SN Ib progenitors (mostly via Case EB mass transfer). All of our models contain a fairly large amount of helium in the envelope $\left(m_{\mathrm{He}}>1.5 \mathrm{M}_{\odot}\right.$; Tables 7, 7 and 7$)$, and the final masses of our models are not large enough to hide this amount of helium in the spectra (cf. Hachinger et al. 2012; Dessart et al. 2012). Therefore no SN Ic is robustly predicted within our grid. The final mass ranges from $3.0 \mathrm{M}_{\odot}$ to $6.6 \mathrm{M}_{\odot}$, which can explain well the ejecta masses of most $\mathrm{SNe} \mathrm{Ib}\left(M_{\text {ejecta }} \simeq 1.0-5.0 \mathrm{M}_{\odot}\right)$ inferred from observations (e.g., Drout et al. 2011; Taddia et al. 2015; Lyman et al. 2016) ${ }^{2}$.

\footnotetext{
2 Note, however, that these ejecta masses have been inferred using a simplified model based on Arnett (1982), which is not very suitable for SNe Ibc (see Dessart et al. 2016).
}

We present the final positions of our SN Ib progenitor models on the HR diagram, in Fig. 10. The final massradius and mass-luminosity relations are also shown in this figure. Unlike SN IIb progenitors, there exists a clear correlation between the final luminosity and the final surface temperature. More luminous (hence more massive) SN Ib progenitors have higher surface temperatures.

This correlation between the final luminosity and surface temperature is reflected by the final mass-radius relation shown in the top-right panel of the figure. The expansion of the helium envelope during the final evolutionary stages becomes more significant for a more compact carbon-oxygen core (the so-called mirror effect). This property of $\mathrm{SN} \mathrm{Ib/c} \mathrm{progenitors} \mathrm{was} \mathrm{first} \mathrm{reported} \mathrm{by}$ Yoon et al. (2010), and our new models confirm their result, even though we adopted a different mass loss rate prescription as discussed in Section 3.3 above. This leads to the following counter-intuitive result that was also discussed by Yoon et al. (2012) in more detail: the brightness in the optical increases as the progenitor mass decreases, while the bolometric luminosity behaves in the opposite way. This is because the bolometric correction increases with increasing $T_{\text {eff }}$ (in this $T_{\text {eff }}$ range).

The expansion of the helium envelope is particularly strong when $M_{\mathrm{f}} \lesssim 3.2 \mathrm{M}_{\odot}$. The predicted optical brightness can reach $M_{\mathrm{V}}=-5.4 \cdots-6.5$ for $M_{\mathrm{f}} \approx 3.0 \mathrm{M}_{\odot}$ (Fig. 10). This may well explain the optical brightness of the progenitor of iPTF13bvn, which was identified by Cao et al. (2013), even without a contribution from its companion, in good agreement with the conclusion of previous studies (Kim et al. 2015; Folatelli et al. 2016; Eldridge \& Maund 2016). However, we need fine-tuned initial masses $\left(M_{\text {init }} \sim 11 \mathrm{M}_{\odot}\right)$ to get this bright progenitor in the optical. More massive progenitors are relatively faint $\left(M_{\mathrm{V}}>-5.0\right)$, and would be more difficult to detect unless they have a brighter companion star. The initial masses of these SN Ib progenitor models are in the range of $11-18 \mathrm{M}_{\odot}$. In our assumption of $\beta=0.2$ and $q=0.9$, the companion star mass does not exceed about $18 \mathrm{M}_{\odot}$, which cannot be much brighter than $M_{\mathrm{V}}=-5.0$ at the pre-SN stage (Kim et al. 2015). Therefore, a sufficiently nearby target would be needed to detect a SN Ib progenitor with $3.5 \mathrm{M}_{\odot} \lesssim M_{\mathrm{f}} \lesssim 7.0 \mathrm{M}_{\odot}$, unless the mass transfer is nearly conservative.

\section{METALLICITY EFFECT}

As shown in Fig. 2, the parameter space for SN Ib progenitors broadens as the metallicity increases. This is because strong winds at a high metallicity during the post mass transfer phase can completely remove the remaining hydrogen layer, for sufficiently short initial orbits. This implies that the SN Ib to SN IIb event rate ratio would increase with increasing metallicity. This prediction seems to be supported by recent analyses on SN 

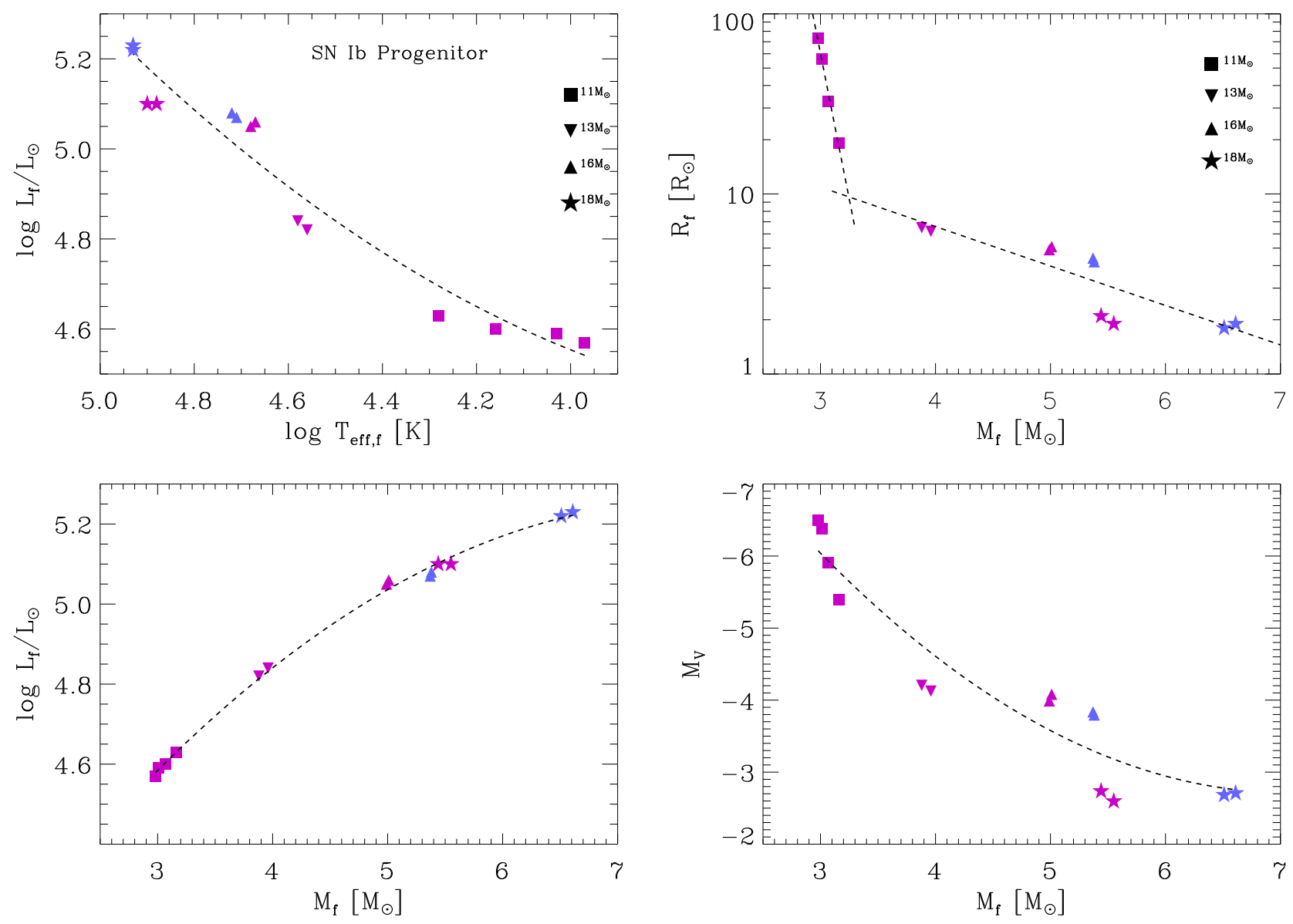

Figure 10. Top left: The final positions of our SN Ib progenitor models on the HR diagram. The different symbols indicate the corresponding initial masses, as indicated by the associated numbers. LMC and solar metallicity models are marked by blue and purple colors, respectively. Top right: The final mass-radius relation of our SN Ib progenitor models. Bottom left: The final mass-luminosity relation of our SN Ib progenitor models. Bottom right: The predicted optical magnitude in $\mathrm{V}$-band of SN Ib progenitors as a function of the final mass.

host galaxies (see Figs. A1 and A2 of Graur et al. 2016; cf.Arcavi et al. 2010), but observational studies still suffer from low-statistics on SNe from metal poor environments. The recent analysis on SN IIP by Dessart et al. (2014) indicates that most SN events occur at around solar metallicity.

A few remarks should be made here. First, given that we still do not have a good constraint on the mass loss rate of stars with stripped envelopes of the relevant mass range as discussed in Section 3.3, our prediction on the metallicity effect is qualitatively sound but quantitatively uncertain. Second, our considered parameter space is limited by certain assumptions chosen for the present study that focuses on stable mass transfer systems (i.e., a fixed mass ratio of $q=0.9$ and a rather low mass accretion efficiency of $\beta=0.2$ ). Although the binary channel with stable mass transfer is the most important path toward SN Ib and SN IIb (Podsiadlowski et al. 1993), the role of non-stable mass transfer needs to be investigated for completeness. Contribution from single stars might also be significant, especially at super-solar metallicity (cf. Meynet et al. 2015; Yoon 2015).

We emphasize that this metallicity effect on the SN $\mathrm{Ib} / \mathrm{SN}$ IIb ratio is largely limited to the case of relatively compact progenitors (SN IIb-B and SN IIb-Y), as clearly implied by Fig. 2. The regime for SN IIb-R is not significantly affected by metallicity. Therefore, a proper investigation on the metallicity effect should distinguish sub-classes of SN IIb.

More specifically, the following predictions on SN binary progenitors can be made with our result of Fig. 2.

- The SN Ib/SN IIb-B and SN Ib/SN IIb-Y ratios would increase more rapidly than the $\mathrm{SN}$ Ib/SN IIb- $\mathrm{R}$ ratio, with increasing metallicity.

- The SN IIb-B/SN IIb-R and SN IIb-Y/SN IIb-R 
ratios would decrease with increasing metallicity.

- In sufficiently metal-poor environments $(Z \approx$ $0.35 Z_{\odot}$ with our fiducial mass loss rate), SN Ib progenitors would be systematically more massive than SN IIb-B/SN IIb-Y progenitors. They would be also more massive than SN Ib progenitors from metal-rich environments, on average.

\section{CONCLUSIONS}

We have presented a new grid of models for SN Ib/IIb progenitors in binary systems. We considered two different metallicities (solar and LMC metallicities) and the initial masses of $10-18 \mathrm{M}_{\odot}$, which correspond to the final masses of about $3.0-8.0 \mathrm{M}_{\odot}$. The initial mass ratio and mass accretion efficiency were fixed to 0.9 and 0.2 , respectively.

Our investigation leads to the following conclusions.

1. In general, a tighter initial orbit leads to stronger stripping of the hydrogen envelope from the primary star. As a result, SN IIb progenitors from Case EB mass transfer in a relatively tight orbit are expected to have compact sizes like SN 2008ax and SN 2011dh progenitors (blue and yellow supergiants; SN IIb-B and SN IIb-Y), while Case LB mass transfer in a wider orbit usually leads to red supergiant progenitors as in the case of SN 1993J and SN 2013df (SN IIb-R; Fig. 2).

2. SN IIb progenitors from Case EB mass transfer can be produced only if mass loss during the post-mass transfer phase is sufficiently weak (Section 3.3; Fig. 2). This means that SN IIb-B and SN IIb$\mathrm{Y}$ progenitors are favored by relatively low initial masses and/or low metallicities. SN IIb-R progenitors are expected for all initial masses and metallicities considered in our study (Fig. 2).

3. The hydrogen envelope mass at the final stage is predicted to be higher than about $M_{\mathrm{H} \text {,env }}=$ $0.15 \mathrm{M}_{\odot}$ in $\mathrm{SN}$ IIb-R progenitors, while SN IIb$\mathrm{Y}$ and SN IIb-B progenitors would have a lower $M_{\mathrm{H} \text {,env }}$ (Fig. 8). This prediction is in good agreement with the previous estimates of $M_{\mathrm{H} \text {,env }} \simeq$ $0.1 \mathrm{M}_{\odot}$ for SN 2011dh $\left(\mathrm{SN}\right.$ IIb-Y), and $M_{\mathrm{H}, \text { env }} \simeq$ $0.2-0.4 \mathrm{M}_{\odot}$ for SN 1993J and SN 2013df (SN IIb$\mathrm{R})$.

4. In our considered mass range, $\mathrm{SN}$ IIb-R progenitors would have $\log L / \mathrm{L}_{\odot} \simeq 4.50-5.30$. The luminosity range of $\mathrm{SN} \mathrm{IIb}-\mathrm{B} / \mathrm{IIb}-\mathrm{Y}$ progenitors is somewhat narrower $\left(\log L / \mathrm{L}_{\odot} \simeq 4.51-5.15\right)$, and most of them (i.e., SN IIb-B/II-Y from Case EB mass transfer) would have $\log L / \mathrm{L}_{\odot}<5.0$.
5. The optical brightness of SN IIb progenitors $\left(M_{\mathrm{V}}<-5.0\right.$ for most cases; Fig. 7) would be systematically much higher than SN Ib progenitors $\left(M_{\mathrm{V}}>-5.0\right.$ for most cases; Fig. 10). For a given initial mass, SN Ib progenitors have higher surface temperatures and lower final masses than SN IIb progenitors.

6. SN Ib progenitors in binary systems are systematically hotter for higher masses. As a result, SN Ib progenitors become brighter in the optical for lower masses, even though the bolometric luminosity increases with increasing mass (Fig. 10). The optically bright progenitor of SN Ib iPTF13bvn $\left(M_{\mathrm{V}} \sim-6\right)$ may be well explained by a progenitor with a final mass of about $3.0 \mathrm{M}_{\odot}$ (Section 5).

7. The impact of wind mass loss after the mass transfer phase becomes more significant at higher metallicity. As a result, the SN Ib to SN IIb event rate ratio would increase with metallicity, while the SN IIb-B/SN IIb-R and SN IIb-Y/SN IIb-R ratios would decrease (Section 6). By contrast, the parameter space for SN IIb-R progenitors is not significantly affected by metallicity for our considered mass range. 


\section{ACKNOWLEDGEMENTS}

This work was supported by the Korea Astronomy and Space Science Institute under the R\&D program (Project No. 3348- 20160002) supervised by the Ministry of Science, ICT and Future Planning. AC acknowledges support from the Ministry of Economy, Development, and Tourism's Millennium Science Initiative through grant IC120009, awarded to The Millennium Institute of Astrophysics, MAS, and from grant Basal CATA PFB 06/09.

\section{REFERENCES}

Aldering, G., Humphreys, R.M., \& Richmond, M. 1994, ApJ, 107, 662

Arcavi, I., Gal-Yam, A., Kasliwal, M.M. et al. 2010,ApJ, 721, 777

Arnett, W.D., ApJ, 253, 785

Ben-Ami, S., Hachinger, S., Gal-Yam, A., et al. 2015, ApJ, 803, 40

Benvenuto, O.G., Bersten, M.C., \& Nomoto, K. 2013, ApJ, 762 74

Bersten, M., Benvenuto, O.G., Nomoto, K., et al. 2012, ApJ, 757, 31

Blinnikov, S.I., Eastman, R., Bartunov, O.S., Popolitov, V.A, \& Woosley, S.E. 1998, ApJ, 496, 454

Bufano, F., Pignata, G., Bersten, M., et al. 2014, MNRAS, 439, 1807

Cao, Y., et al. 2013, ApJ, 775, L7

Chevalier, R.A., \& Soderberg, A. 2010, ApJ, 711, 40

Chornock, R., Filippenko, A.V., \& Li, W. et al. 2011, ApJ, 739, 41

Claeys, J.S.W., de Mink, S.E., Pols, O.R., Eldridge, J.J., Baes, M. 2011, A\&A, 528, A131

De Loore, C.W.H., \& Doom, C. 1992, Structure and evolution of single and binary stars, Dordrecht, Boston: Kluwer Academic Publishers, Astrophysics and space science library, vol. 179

Dessart, L., Gutierrez, C.P., \& Hamuy, M., et al. 2014, MNRAS, 440, 1856

Dessart, L., \& Hillier, D.J. 2011, MNRAS, 415, 3497

Dessart, L., Hillier, D.J., Livne, E., Yoon, S.-C., Woosley, S., Waldman, R., Langer, N. 2011, MNRAS, 414, 2985

Dessart, L., Hillier, D.J., Li, C., Woosley, S. 2012, MNRAS, 424, 2139

Dessart, L., Hillier, D.J., Woosley, S., Livne, E., Waldman, R., Yoon, S.-C., \& Langer, N. 2015, MNRAS, 453, 2189

Dessart, L., Hillier, D.J., Woosley, S., Livne, E., Waldman, R., Yoon, S.-C., \& Langer, N. 2016, MNRAS, 458, 1618

Drout, M.R., Massey, Ph., Meynet, G., Tokarz, S., \& Caldwell, N. 2009, ApJ, 703, 441

Drout, M.R., Soderberg, A.M, Gal-Yam, A., et al. 2011, ApJ, 741,97

Eggleton, P. 2011, Evolutionary Processes in Binary and Multiple Stars, by Peter Eggleton, Cambridge, UK: Cambridge University Press

Eldridge, J.J., Fraser, M., Smartt, S.J., Mound, J.R., \& Crockett, R. M. 2013, MNRAS, 436, 774

Eldridge, J.J., Fraser, M., Mound, J.R., \& Smartt, S.J. 2015, MNRAS, 446, 2689

Eldridge, J.J., \& Maund, J.R. 2016, MNRAS, 461, L117

Ensman, L. M., \& Woosley, S.E. 1988, ApJ, 333, 754

Ergon, M., Jerkstrand, A., Sollerman, J., et al. 2015, A\&A, 580, A142

Folatelli, G., Bersten, M.C., Kuncarayakti, H., Benvenuto, O., Maeda, K., \& Nomoto, K. 2015, ApJ, 811, 147

Folatelli, G., Van Dyk, S.D., Kuncarayakt, D., et al. 2016, ApJ, 825, L22
Graur, O., Banco, F., Modjaz, M., Shvvers, I., Filippenko, A.V., Li, W., \& Smith, N. 2016, [arXiv:1609.02923]

Hachinger, S., Mazzaili, P.A., Taubenberger, S., Hillebrandt, W., Nomoto, K., \& Sauer, D.N. 2012, MNRAS, 422, 70

Höflich, P. 1995, ApJ, 440, 821

Kolb, U., \& Ritter, H. 1990, A\&A, 236, 385

Kilpatrick, C.D., Foley, R.J., Abramson, L.E., et al. 2016, submitted to MNRAS, [arXiv:1610.04587]

Kim, H.-J., Yoon, S.-C., \& Koo, B.-C. 2015, ApJ, 809, 131

Kramble, A., Margutti, R., Soderberg, A.M., et al. 2016, ApJ, 818,111

Kumar, B., Pandey, S.B., Sahu, D.K., et al. 2013, MNRAS, 431, 308

Langer, N. 2012, ARA\&A, 50, 107

Lyman, J., Bersier, D., James, P.A., Mazzali, P.A., Eldridge, J.J., Fraser, M., \& Pian, E. 2016, MNRAS, 457, 328

Maeda, K., Katsuda, S., Bamba, A., Terada, Y., \& Fukazawa, Y. 2014, ApJ, 785, 95

Maeda, K., Hattori, T., Milisavljevic, D., et al. 2015, ApJ, 807, 35

Mahuerhan, J.C., Williams, G.G. \& Leonard, D.C. et al. 2015, MNRAS, 453, 4467

Maund, J.R., Fraser, M., Ergon, M. et al. 2011, ApJL, 739, L37

Maund, J.R., Smartt, S.J., Kudritzki, R.P., Podsiadlowski, Ph., \& Gilmore, G.F. 2004, Nature, 427, 129

Maund, J.R., Wheeler, J.C., Patat, F., Wang, L., Baade, D., \& Höflich, P.A. 2007, ApJ, 671, 1944

Meynet, G., Chomienne, V., \& Ekström et al. 2015, A\&A, 575, A60

Milisavljevic, D., Margutti, R., Soderberg, A.M., et al. 2013, ApJ, 767,71

Morozova, V., Piro, A.L., Renzo, M., \& Ott, C.D. 2015, ApJ, 814, 63

Nakar, E., \& Piro, A.L. 2014, ApJ, 788, 193

Nugis, T., \& Lamers, H.J.G.L.M. 2000, A\&A, 360, 227

Pastetter, L., \& Ritter, H. 1989, A\&A, 214, 186

Paxton, B., Bildsten, L., Dotter, A., Herwig, F., Lesaffre, P., \& Timmes, F. 2011, ApJS, 192, 3

Paxton, B., Cantiello, M., Arras, Ph., et al. 2013, ApJS, 208, 4

Paxton, B., Marchant, P., Schwab, J., et al. 2015, ApJS, 220, 15

Pastorello, A., Kasliwal, M.M., Crockett, R.M., et al. 2008,

MNRAS, 389, 955

Petrovic, J., Langer, N., \& van der Hucht, K.A. 2005, A\&A, 435, 1013

Podsiadlowski, Ph., Hsu, J.J.L., Joss, P.C., \& Ross, R.R., 1993, Nature, 364, 509

Qiu, Y., Li, W., Qiao, Q., \& Hu, J. 1999, ApJ, 117, 736

Reilly, E., Maund, J.R., Baade, D., et al. 2016, MNRAS, 45, 288

Ritter, H. 1988, A\&A, 202, 93

Shigeyama, T., Suzuki, T., Kumagai, S., Nomoto, K., Saio, H., \& Yamaoka, H. 1994, ApJ, 420, 341

Shivvers, I., Modjaz, M., \& Zheng, W. et al. 2016, PASP, submitted, [arXiv:1609.02922]

Smartt, S.J. 2015, PASA, 32, 16

Smith, N., Li, W., Filippenko, A.V., \& Chornock, R. 2011, MNRAS, 412, 1522

Stancliffe, R., \& Eldridge, J.J. 2009, MNRAS, 396, 1699

Stevance, H.F., Maund, J.R., \& Baade, D. et al. 2016, MNRAS, 461, 2019

Szalai, T., Vinkó, J., Nagy, A.P., et al. 2016, MNRAS, 460, 1500

Taddia, F., Sollerman, J., Leloudas, G., et al. 2015, A\&A, 574, A60

Tartaglia, L., Fraser, M., Sand, D.J., et al. 2016, submitted to ApJL, [arXiv:1611.00419]

Taubenberger, S., Navasardyan, H., Maurer, J.I., et al. 2011, MNRAS, 413, 2140

Tran, H.D., Filippenko, A.V., Schmidt, G.D., Bjorkman, K.S., Jannuzi, B.T., \& Smith, P.S. 1997, PASP, 109, 489 
Table 1. Physical properties of the final models of the primary star at $Z=0.007$.

\begin{tabular}{|c|c|c|c|c|c|c|c|c|c|c|c|c|c|c|c|}
\hline Name & $M_{\mathrm{f}}$ & $L_{\mathrm{f}}$ & $R_{\mathrm{f}}$ & $T_{\text {eff }, \mathrm{f}}$ & $\mathrm{He}$ & $\mathrm{CO}$ & Henv & $m_{\mathrm{H}}$ & $m_{\mathrm{He}}$ & $Y_{\mathrm{s}, \mathrm{f}}$ & $\dot{M}_{\mathrm{tr}}$ & $\dot{M}_{\mathrm{w}}$ & $f_{\mathrm{RL}}$ & Case & SN \\
\hline Lm10p20 & 2.99 & 4.58 & 66.6 & 4.00 & 2.944 & 1.509 & 0.046 & 0.005 & 1.440 & 0.78 & -12.67 & -6.75 & -0.15 & $\mathrm{EBB}$ & IIb-B \\
\hline Lm10p50 & 3.01 & 4.61 & 143.1 & 3.84 & 2.955 & 1.511 & 0.055 & 0.007 & 1.448 & 0.76 & -5.51 & -6.44 & -0.01 & $\mathrm{EBB}$ & IIb-Y \\
\hline Lm10p150 & 3.07 & 4.61 & 278.8 & 3.69 & 2.979 & 1.527 & 0.090 & 0.015 & 1.486 & 0.73 & -5.33 & -6.19 & -0.04 & $\mathrm{EBB}$ & IIb-Y \\
\hline Lm10p300 & 3.33 & 4.65 & 387.4 & 3.63 & 3.147 & 1.602 & 0.182 & 0.048 & 1.622 & 0.57 & -6.44 & -6.02 & -0.09 & $\mathrm{EBB}$ & IIb-Y \\
\hline Lm10p700 & 3.70 & 4.69 & 529.6 & 3.57 & 3.311 & 1.676 & 0.392 & 0.205 & 1.745 & 0.38 & -6.04 & -5.85 & -0.11 & LBB & IIb-R \\
\hline Lm10p800 & 3.90 & 4.69 & 560.9 & 3.56 & 3.320 & 1.681 & 0.579 & 0.336 & 1.802 & 0.34 & -5.86 & -5.83 & -0.11 & LBB & IIb-R \\
\hline Lm10p900 & 4.18 & 4.69 & 583.2 & 3.55 & 3.335 & 1.686 & 0.843 & 0.526 & 1.889 & 0.31 & -5.71 & -5.81 & -0.11 & $\mathrm{LBB}$ & IIb-R \\
\hline Lm10p1000 & 4.81 & 4.70 & 603.2 & 3.55 & 3.378 & 1.701 & 1.432 & 0.951 & 2.070 & 0.29 & -5.45 & -5.79 & -0.09 & $\mathrm{LBB}$ & IIb-R \\
\hline Lm11p10 & 3.53 & 4.72 & 10.0 & 4.44 & 3.417 & 1.748 & 0.110 & 0.010 & 1.683 & 0.85 & -99.00 & -6.11 & -0.79 & $\mathrm{~EB}$ & IIb-B \\
\hline Lm11p20 & 3.56 & 4.73 & 61.1 & 4.05 & 3.444 & 1.762 & 0.119 & 0.017 & 1.697 & 0.67 & -11.13 & -6.28 & -0.17 & $\mathrm{~EB}$ & IIb-B \\
\hline Lm11p30 & 3.57 & 4.73 & 99.3 & 3.95 & 3.452 & 1.766 & 0.122 & 0.019 & 1.700 & 0.67 & -5.36 & -6.40 & 0.03 & $\mathrm{EBB}$ & IIb-B \\
\hline Lm11p50 & 3.59 & 4.73 & 135.9 & 3.88 & 3.466 & 1.772 & 0.120 & 0.021 & 1.702 & 0.67 & -5.39 & -6.29 & 0.01 & $\mathrm{EBB}$ & IIb-B \\
\hline Lm11p100 & 3.60 & 4.73 & 206.3 & 3.79 & 3.475 & 1.778 & 0.124 & 0.024 & 1.706 & 0.67 & -5.87 & -6.13 & -0.02 & $\mathrm{EBB}$ & IIb-Y \\
\hline Lm11p200 & 3.62 & 4.74 & 290.9 & 3.71 & 3.489 & 1.784 & 0.131 & 0.028 & 1.717 & 0.67 & -10.39 & -6.01 & -0.12 & $\mathrm{~EB}$ & IIb-Y \\
\hline Lm11p300 & 3.67 & 4.74 & 334.7 & 3.68 & 3.526 & 1.802 & 0.141 & 0.035 & 1.738 & 0.67 & -15.88 & -5.95 & -0.22 & $\mathrm{~EB}$ & IIb-Y \\
\hline Lm11p500 & 3.93 & 4.77 & 487.8 & 3.61 & 3.664 & 1.880 & 0.263 & 0.089 & 1.848 & 0.49 & -7.13 & -5.77 & -0.12 & $\mathrm{LBB}$ & IIb-R \\
\hline Lm11p700 & 4.08 & 4.79 & 561.6 & 3.58 & 3.741 & 1.919 & 0.341 & 0.160 & 1.888 & 0.40 & -5.92 & -5.70 & -0.11 & $\mathrm{LBB}$ & IIb-R \\
\hline Lm11p1000 & 4.45 & 4.79 & 630.6 & 3.56 & 3.787 & 1.988 & 0.660 & 0.388 & 1.991 & 0.33 & -5.66 & -5.65 & -0.11 & $\mathrm{LBB}$ & IIb-R \\
\hline Lm11p1200 & 5.03 & 4.80 & 668.6 & 3.55 & 3.811 & 1.957 & 1.220 & 0.793 & 2.146 & 0.29 & -5.36 & -5.62 & -0.09 & $\mathrm{LBB}$ & IIL/IIP \\
\hline Lm13p20 & 4.42 & 4.90 & 12.6 & 4.44 & 4.295 & 2.273 & 0.123 & 0.017 & 1.958 & 0.72 & -99.00 & -6.00 & -0.82 & $\mathrm{~EB}$ & IIb-B \\
\hline Lm13p50 & 4.42 & 4.90 & 13.8 & 4.42 & 4.299 & 2.276 & 0.124 & 0.017 & 1.972 & 0.72 & -99.00 & -6.01 & -0.89 & $\mathrm{~EB}$ & IIb-B \\
\hline Lm13p100 & 4.43 & 4.90 & 14.6 & 4.41 & 4.303 & 2.278 & 0.125 & 0.018 & 1.972 & 0.71 & -99.00 & -6.01 & -0.93 & $\mathrm{~EB}$ & IIb-B \\
\hline Lm13p300 & 4.46 & 4.91 & 19.1 & 4.35 & 4.329 & 2.295 & 0.131 & 0.021 & 1.981 & 0.71 & -99.00 & -6.00 & -0.96 & $\mathrm{~EB}$ & IIb-B \\
\hline Lm13p400 & 4.56 & 4.92 & 31.0 & 4.25 & 4.409 & 2.348 & 0.149 & 0.029 & 2.010 & 0.71 & -99.00 & -5.99 & -0.94 & $\mathrm{~EB}$ & IIb-B \\
\hline Lm13p500 & 4.80 & 4.94 & 350.6 & 3.72 & 4.521 & 2.415 & 0.275 & 0.066 & 2.128 & 0.70 & -37.90 & -5.66 & -0.39 & $\mathrm{~EB}$ & IIb-Y \\
\hline Lm13p600 & contact & & & & & & & & & & & & & & \\
\hline Lm13p900 & contact & & & & & & & & & & & & & & \\
\hline Lm13p1000 & 5.06 & 4.97 & 637.4 & 3.60 & 4.691 & 2.524 & 0.370 & 0.141 & 2.191 & 0.47 & -7.33 & -5.41 & -0.13 & $\mathrm{LBB}$ & IIb-R \\
\hline Lm13p1100 & 5.20 & 4.97 & 690.3 & 3.58 & 4.723 & 2.543 & 0.479 & 0.223 & 2.231 & 0.42 & -5.21 & -5.38 & -0.08 & $\mathrm{LBB}$ & IIb-R \\
\hline Lm13p1300 & 5.35 & 4.97 & 726.7 & 3.57 & 4.750 & 2.563 & 0.599 & 0.311 & 2.279 & 0.38 & -5.02 & -5.35 & -0.08 & $\mathrm{LBB}$ & IIb-R \\
\hline Lm13p1500 & 5.87 & 4.98 & 794.0 & 3.56 & 4.774 & 2.578 & 1.097 & 0.667 & 2.417 & 0.32 & -4.72 & -5.31 & -0.08 & $\mathrm{LBB}$ & IIb-R \\
\hline Lm16p50 & 5.37 & 5.07 & 4.4 & 4.71 & 5.368 & 3.143 & 0.000 & 0.000 & 2.041 & 0.99 & -99.00 & -5.55 & -0.97 & $\mathrm{~EB}$ & $\mathrm{Ib}$ \\
\hline Lm16p100 & 5.38 & 5.08 & 4.2 & 4.72 & 5.383 & 3.173 & 0.000 & 0.000 & 2.023 & 0.99 & -99.00 & -5.54 & -0.98 & $\mathrm{~EB}$ & $\mathrm{Ib}$ \\
\hline Lm16p200 & contact & & & & & & & & & & & & & & \\
\hline Lm16p500 & contact & & & & & & & & & & & & & & \\
\hline Lm16p1800 & contact & & & & & & & & & & & & & & \\
\hline Lm16p1900 & contact & & & & & & & & & & & & & & \\
\hline Lm16p2000 & 6.35 & 5.15 & 713.3 & 3.62 & 6.056 & 3.774 & 0.292 & 0.074 & 2.277 & 0.57 & -24.51 & -5.12 & -0.37 & $\mathrm{LB}$ & IIb-R \\
\hline Lm16p2400 & 6.48 & 5.15 & 865.3 & 3.58 & 6.086 & 3.657 & 0.389 & 0.129 & 2.491 & 0.52 & -13.10 & -5.05 & -0.25 & $\mathrm{LB}$ & IIb-R \\
\hline Lm16p2600 & 7.12 & 5.16 & 1098.3 & 3.53 & 6.116 & 3.679 & 1.005 & 0.553 & 2.678 & 0.37 & -4.51 & -4.95 & -0.07 & $\mathrm{LBB}$ & IIL/IIP \\
\hline Lm18p50 & 6.51 & 5.22 & 1.8 & 4.93 & 6.508 & 4.221 & 0.000 & 0.000 & 2.116 & 0.99 & -99.00 & -5.36 & -0.99 & $\mathrm{~EB}$ & $\mathrm{Ib}$ \\
\hline Lm18p300 & 6.61 & 5.23 & 1.9 & 4.93 & 6.614 & 4.276 & 0.000 & 0.000 & 2.172 & 0.99 & -99.00 & -5.35 & -1.00 & $\mathrm{~EB}$ & $\mathrm{Ib}$ \\
\hline Lm18p400 & contact & & & & & & & & & & & & & & \\
\hline Lm18p1700 & contact & & & & & & & & & & & & & & \\
\hline Lm18p1900 & contact & & & & & & & & & & & & & & \\
\hline Lm18p2000 & 7.73 & 5.29 & 710.8 & 3.66 & 7.311 & 4.707 & 0.415 & 0.111 & 2.707 & 0.59 & -26.18 & -4.93 & -0.36 & LB & IIb-R \\
\hline Lm18p2100 & 7.76 & 5.29 & 744.4 & 3.65 & 7.328 & 4.722 & 0.432 & 0.120 & 2.718 & 0.59 & -23.33 & -4.91 & -0.34 & LB & IIb-R \\
\hline Lm18p2200 & 7.79 & 5.29 & 769.8 & 3.64 & 7.348 & 4.738 & 0.443 & 0.127 & 2.731 & 0.59 & -21.32 & -4.90 & -0.32 & $\mathrm{LB}$ & IIb-R \\
\hline Lm18p2300 & 7.81 & 5.30 & 787.0 & 3.64 & 7.363 & 4.749 & 0.449 & 0.131 & 2.730 & 0.59 & -20.02 & -4.89 & -0.30 & $\mathrm{LB}$ & IIb-R \\
\hline Lm18p2400 & 7.84 & 5.30 & 806.4 & 3.63 & 7.380 & 4.761 & 0.462 & 0.138 & 2.747 & 0.59 & -18.70 & -4.87 & -0.29 & $\mathrm{LB}$ & IIb-R \\
\hline Lm18p2500 & 8.46 & 5.30 & 1039.8 & 3.58 & 7.411 & 4.784 & 1.047 & 0.528 & 2.935 & 0.39 & -4.84 & -4.78 & -0.07 & LBB & IIL/IIP \\
\hline
\end{tabular}

Note- $M_{\mathrm{f}}$ : total mass in units of $\mathrm{M}_{\odot}, L_{\mathrm{f}}$ : luminosity in units of $\mathrm{L}_{\odot}$ in logarithmic scale, $R_{\mathrm{f}}:$ radius in units of $\mathrm{R}_{\odot}, T_{\mathrm{eff}}:$ surface temperature in units of $\mathrm{K}$ in logarithmic scale, He: He core mass, $\mathrm{CO}$ : carbon-oxygen core mass, $\mathrm{H}_{\text {env }}$ : mass of the hydrogen envelope, $m_{\mathrm{H}}$ : total mass of hydrogen (i.e., $\left.m_{\mathrm{H}}=\int X_{\mathrm{H}} d M_{r}\right), m_{\mathrm{He}}$ : total mass of helium, $Y_{\mathrm{s}, \mathrm{f}}$ : mass fraction of helium at the surface, $\dot{M}_{\mathrm{tr}}$ : mass transfer rate in units of $\mathrm{M}_{\odot} \mathrm{yr}^{-1}$ in logarithmic scale, $\dot{M}_{w}$ : wind mass loss rate in units of $\mathrm{M}_{\odot} \mathrm{yr}^{-1}$ in logarithmic scale, $f_{\mathrm{RL}}$ : Roche-lobe filling factor. 
Table 2. Physical properties of the final models of the primary star at $Z=0.02$.

\begin{tabular}{|c|c|c|c|c|c|c|c|c|c|c|c|c|c|c|c|}
\hline Name & $M_{\mathrm{f}}$ & $L_{\mathrm{f}}$ & $R_{\mathrm{f}}$ & $T_{\text {eff }, \mathrm{f}}$ & $\mathrm{He}$ & $\mathrm{CO}$ & Henv & $m_{\mathrm{H}}$ & $m_{\mathrm{He}}$ & $Y_{\mathrm{s}, \mathrm{f}}$ & $\dot{M}_{\mathrm{tr}}$ & $\dot{M}_{\mathrm{w}}$ & $f_{\mathrm{RL}}$ & Case & SN \\
\hline Sm10p50 & 2.04 & 4.66 & 258.5 & 3.72 & 2.041 & 1.360 & 0.000 & 0.000 & 0.660 & 0.97 & -1.52 & -6.16 & 0.12 & $\mathrm{EBB}$ & - \\
\hline Sm10p200 & 2.73 & 4.43 & 139.0 & 3.80 & 2.726 & 1.391 & 0.000 & 0.000 & 1.294 & 0.98 & -99.00 & -6.69 & -0.64 & EB & - \\
\hline Sm10p400 & 3.12 & 4.52 & 429.0 & 3.57 & 2.976 & 1.501 & 0.142 & 0.047 & 1.507 & 0.54 & -11.29 & -6.16 & -0.20 & LB & IIb-R \\
\hline Sm10p600 & 3.29 & 4.59 & 532.2 & 3.55 & 3.084 & 1.541 & 0.211 & 0.110 & 1.573 & 0.40 & -7.51 & -5.98 & -0.15 & LB & IIb-R \\
\hline Sm10p800 & 3.55 & 4.61 & 598.5 & 3.53 & 3.105 & 1.554 & 0.444 & 0.262 & 1.656 & 0.34 & -6.54 & -5.91 & -0.14 & LB & IIb-R \\
\hline Sm10p1000 & 4.04 & 4.61 & 633.9 & 3.51 & 3.116 & 1.560 & 0.926 & 0.588 & 1.808 & 0.31 & -6.33 & -5.89 & -0.13 & & \\
\hline Sm11p20 & 2.98 & 4.57 & 73.0 & 3.97 & 2.978 & 1.528 & 0.000 & 0.000 & 1.391 & 0.98 & -35.55 & -6.73 & -0.15 & EB & $\mathrm{Ib}$ \\
\hline Sm11p50 & 3.01 & 4.59 & 56.3 & 4.03 & 3.005 & 1.539 & 0.000 & 0.000 & 1.404 & 0.98 & -99.00 & -6.04 & -0.64 & $\mathrm{~EB}$ & $\mathrm{Ib}$ \\
\hline Sm11p200 & 3.07 & 4.60 & 32.5 & 4.16 & 3.069 & 1.563 & 0.000 & 0.000 & 1.438 & 0.98 & -99.00 & -5.94 & -0.92 & EB & $\mathrm{Ib}$ \\
\hline Sm11p300 & 3.16 & 4.63 & 19.2 & 4.28 & 3.158 & 1.598 & 0.000 & 0.000 & 1.487 & 0.98 & -99.00 & -5.90 & -0.96 & $\mathrm{~EB}$ & $\mathrm{Ib}$ \\
\hline Sm11p400 & 3.47 & 4.68 & 458.4 & 3.60 & 3.330 & 1.679 & 0.141 & 0.033 & 1.664 & 0.67 & -11.29 & -5.92 & -0.17 & LB & IIb-R \\
\hline Sm11p500 & 3.55 & 4.69 & 511.9 & 3.58 & 3.387 & 1.709 & 0.166 & 0.052 & 1.689 & 0.53 & -8.58 & -5.86 & -0.15 & LB & IIb-R \\
\hline Sm11p600 & 3.66 & 4.70 & 565.4 & 3.56 & 3.459 & 1.743 & 0.198 & 0.082 & 1.719 & 0.46 & -6.76 & -5.81 & -0.12 & LB & IIb-R \\
\hline Sm11p700 & 3.71 & 4.71 & 586.9 & 3.56 & 3.497 & 1.765 & 0.212 & 0.096 & 1.732 & 0.41 & -6.57 & -5.78 & -0.13 & LB & IIb-R \\
\hline Sm11p800 & 3.79 & 4.75 & 642.2 & 3.55 & 3.544 & 1.888 & 0.246 & 0.127 & 1.636 & 0.37 & -5.79 & -5.69 & -0.11 & LBB & IIb-R \\
\hline Sm11p1000 & 4.04 & 4.72 & 675.4 & 3.53 & 3.547 & 1.790 & 0.495 & 0.285 & 1.840 & 0.34 & -6.08 & -5.72 & -0.12 & LBB & IIb-R \\
\hline Sm11p1200 & 4.39 & 4.76 & 743.2 & 3.52 & 3.575 & 1.903 & 0.818 & 0.507 & 1.831 & 0.31 & -5.30 & -5.63 & -0.10 & LBB & IIb-R \\
\hline Sm11p1400 & 5.62 & 4.73 & 732.9 & 3.51 & 3.606 & 1.816 & 2.018 & 1.316 & 2.323 & 0.30 & -5.75 & -5.67 & -0.09 & LBB & IIL/IIP \\
\hline Sm13p50 & 3.88 & 4.82 & 6.5 & 4.56 & 3.878 & 2.095 & 0.000 & 0.000 & 1.627 & 0.98 & -99.00 & -5.65 & -0.95 & $\mathrm{~EB}$ & $\mathrm{Ib}$ \\
\hline Sm13p500 & 3.96 & 4.84 & 6.2 & 4.58 & 3.957 & 2.144 & 0.000 & 0.000 & 1.645 & 0.98 & -99.00 & -5.63 & -0.99 & EB & $\mathrm{Ib}$ \\
\hline Sm13p600 & contact & & & & & & & & & & & & & & \\
\hline Sm13p700 & contact & & & & & & & & & & & & & & \\
\hline Sm13p1100 & contact & & & & & & & & & & & & & & \\
\hline Sm13p1150 & contact & & & & & & & & & & & & & & \\
\hline Sm13p1200 & 4.86 & 4.93 & 743.2 & 3.56 & 4.567 & 2.419 & 0.288 & 0.133 & 2.078 & 0.38 & -8.04 & -5.40 & -0.17 & LB & IIb-R \\
\hline Sm13p1500 & 5.12 & 4.94 & 828.3 & 3.54 & 4.618 & 2.461 & 0.505 & 0.278 & 2.152 & 0.34 & -5.67 & -5.35 & -0.11 & LB & IIb-R \\
\hline Sm13p1700 & 5.33 & 4.94 & 866.6 & 3.53 & 4.629 & 2.461 & 0.699 & 0.407 & 2.225 & 0.33 & -5.28 & -5.33 & -0.10 & LB & IIb-R \\
\hline Sm13p2000 & 6.08 & 4.95 & 933.4 & 3.51 & 4.666 & 2.487 & 1.411 & 0.890 & 2.447 & 0.30 & -4.85 & -5.29 & -0.09 & LB & IIL/IIP \\
\hline Sm16p50 & 4.99 & 5.05 & 4.9 & 4.68 & 4.994 & 3.182 & 0.000 & 0.000 & 1.659 & 0.98 & -99.00 & -5.35 & -0.96 & EB & $\mathrm{Ib}$ \\
\hline Sm16p300 & 5.01 & 5.06 & 5.1 & 4.67 & 5.009 & 3.208 & 0.000 & 0.000 & 1.649 & 0.98 & -99.00 & -5.34 & -0.99 & EB & $\mathrm{Ib}$ \\
\hline Sm16p400 & contact & & & & & & & & & & & & & & \\
\hline Sm16p1400 & contact & & & & & & & & & & & & & & \\
\hline Sm16p1600 & contact & & & & & & & & & & & & & & \\
\hline Sm16p1700 & 6.08 & 5.14 & 3.2 & 4.79 & 6.056 & 3.644 & 0.024 & 0.001 & 2.250 & 0.94 & -99.00 & -5.27 & -1.00 & LB & IIb-B \\
\hline Sm16p1800 & 6.19 & 5.16 & 27.5 & 4.33 & 6.098 & 3.678 & 0.096 & 0.011 & 2.293 & 0.76 & -99.00 & -5.40 & -0.98 & LB & IIb-B \\
\hline Sm16p1900 & 6.43 & 5.12 & 364.4 & 3.76 & 6.157 & 3.700 & 0.273 & 0.073 & 2.496 & 0.62 & -99.00 & -5.39 & -0.67 & $\mathrm{LB}$ & IIb-Y \\
\hline Sm16p2000 & 6.42 & 5.18 & 748.1 & 3.62 & 6.189 & 3.725 & 0.231 & 0.059 & 2.418 & 0.62 & -24.11 & -5.07 & -0.35 & LB & IIb-R \\
\hline Sm16p2200 & 6.48 & 5.15 & 717.3 & 3.62 & 6.214 & 3.746 & 0.262 & 0.074 & 2.470 & 0.62 & -28.71 & -5.12 & -0.39 & LB & IIb-R \\
\hline Sm16p2400 & 6.53 & 5.18 & 822.2 & 3.60 & 6.267 & 3.893 & 0.262 & 0.078 & 2.300 & 0.62 & -19.33 & -5.03 & -0.31 & LB & IIb-R \\
\hline Sm16p2600 & 6.76 & 5.18 & 974.9 & 3.56 & 6.285 & 3.805 & 0.479 & 0.204 & 2.531 & 0.46 & -8.91 & -4.97 & -0.18 & $\mathrm{LB}$ & IIb-R \\
\hline Sm16p2700 & 7.33 & 5.18 & 1121.5 & 3.53 & 6.307 & 3.815 & 1.021 & 0.575 & 2.693 & 0.36 & -4.42 & -4.91 & -0.07 & LBB & IIL/IIP \\
\hline Sm18p50 & 5.44 & 5.10 & 2.1 & 4.88 & 5.440 & 3.478 & 0.000 & 0.000 & 1.571 & 0.98 & -99.00 & -5.29 & -0.98 & EB & $\mathrm{Ib}$ \\
\hline Sm18p500 & 5.55 & 5.10 & 1.9 & 4.90 & 5.548 & 3.536 & 0.000 & 0.000 & 1.613 & 0.98 & -99.00 & -5.29 & -1.00 & $\mathrm{~EB}$ & $\mathrm{Ib}$ \\
\hline Sm18p600 & contact & & & & & & & & & & & & & & \\
\hline Sm18p1000 & contact & & & & & & & & & & & & & & \\
\hline Sm18p1900 & contact & & & & & & & & & & & & & & \\
\hline Sm18p2000 & 6.62 & 5.19 & 1.7 & 4.94 & 6.624 & 4.330 & 0.000 & 0.000 & 2.158 & 0.98 & -99.00 & -5.18 & -1.00 & LB & $\mathrm{Ib}$ \\
\hline Sm18p2200 & 7.04 & 5.16 & 1.7 & 4.93 & 6.953 & 4.411 & 0.084 & 0.007 & 2.526 & 0.81 & -99.00 & -5.36 & -1.00 & $\mathrm{LB}$ & IIb-B \\
\hline $\operatorname{Sm} 18 p 2400$ & 7.28 & 5.26 & 710.3 & 3.65 & 7.047 & 4.458 & 0.231 & 0.050 & 2.565 & 0.67 & -41.32 & -4.97 & -0.46 & LB & IIb-R \\
\hline Sm18p2800 & 7.37 & 5.27 & 794.0 & 3.63 & 7.126 & 4.623 & 0.243 & 0.058 & 2.475 & 0.67 & -32.79 & -4.92 & -0.41 & $\mathrm{LB}$ & IIb-R \\
\hline Sm18p3000 & 7.43 & 5.27 & 818.4 & 3.62 & 7.170 & 4.594 & 0.264 & 0.066 & 2.561 & 0.67 & -30.71 & -4.91 & -0.39 & LB & IIb-R \\
\hline Sm18p3100 & 7.92 & 5.27 & 1140.1 & 3.55 & 7.203 & 4.585 & 0.720 & 0.349 & 2.767 & 0.37 & -7.41 & -4.79 & -0.15 & LB & IIb-R \\
\hline
\end{tabular}


Table 3. Physical properties of the final models of the primary star at $Z=0.02$ with a reduced mass loss rate $(\eta=0.48)$

\begin{tabular}{l|ccccccccccccccc}
\hline \hline \multicolumn{1}{c}{ Name } & $M_{\mathrm{f}}$ & $L_{\mathrm{f}}$ & $R_{\mathrm{f}}$ & $T_{\mathrm{eff}, \mathrm{f}}$ & $\mathrm{He}$ & $\mathrm{CO}$ & Henv & $m_{\mathrm{H}}$ & $m_{\mathrm{He}}$ & $Y_{\mathrm{s}, \mathrm{f}}$ & $\dot{M}_{\mathrm{tr}}$ & $\dot{M}_{\mathrm{w}}$ & $f_{\mathrm{RL}}$ & Case & SN \\
\hline Tm11p20 & 3.21 & 4.63 & 87.4 & 3.95 & 3.161 & 1.591 & 0.044 & 0.004 & 1.534 & 0.81 & -5.27 & -6.91 & 0.02 & EBB & IIb-B \\
Tm11p100 & 3.27 & 4.64 & 237.4 & 3.73 & 3.195 & 1.611 & 0.074 & 0.009 & 1.567 & 0.77 & -5.62 & -6.53 & -0.03 & EBB & IIb-Y \\
Tm11p200 & 3.33 & 4.65 & 314.9 & 3.67 & 3.231 & 1.629 & 0.097 & 0.015 & 1.603 & 0.76 & -14.33 & -6.42 & -0.17 & EB & IIb-R \\
Tm11p300 & 3.40 & 4.67 & 395.7 & 3.63 & 3.290 & 1.661 & 0.110 & 0.021 & 1.628 & 0.71 & -12.72 & -6.31 & -0.18 & LB & IIb-R \\
Tm11p400 & 3.58 & 4.70 & 503.0 & 3.59 & 3.408 & 1.720 & 0.173 & 0.049 & 1.707 & 0.59 & -6.76 & -6.18 & -0.10 & LBB & IIb-R \\
Tm11p500 & contact & & & & & & & & & & & & & & \\
\hline Tm13p20 & 4.26 & 4.87 & 8.6 & 4.51 & 4.218 & 2.183 & 0.044 & 0.002 & 1.887 & 0.87 & -99.00 & -5.99 & -0.89 & EB & IIb-B \\
Tm13p50 & 4.27 & 4.87 & 8.6 & 4.51 & 4.230 & 2.191 & 0.043 & 0.002 & 1.888 & 0.87 & -99.00 & -5.99 & -0.94 & EB & IIb-B \\
Tm13p100 & 4.28 & 4.87 & 8.7 & 4.51 & 4.234 & 2.193 & 0.044 & 0.002 & 1.895 & 0.87 & -99.00 & -5.99 & -0.96 & EB & IIb-B \\
Tm13p300 & 4.32 & 4.88 & 8.8 & 4.51 & 4.270 & 2.218 & 0.047 & 0.003 & 1.908 & 0.86 & -99.00 & -5.99 & -0.98 & EB & IIb-B \\
Tm13p500 & contact & & & & & & & & & & & & & \\
\hline Tm16p50 & 5.55 & 5.09 & 4.6 & 4.71 & 5.548 & 3.242 & 0.000 & 0.000 & 2.095 & 0.98 & -99.00 & -5.62 & -0.97 & EB & Ib \\
Tm16p100 & 5.57 & 5.09 & 4.6 & 4.70 & 5.572 & 3.253 & 0.000 & 0.000 & 2.110 & 0.98 & -99.00 & -5.62 & -0.98 & EB & Ib \\
\hline
\end{tabular}

Table 4. Properties of the identified SN IIb progenitors

\begin{tabular}{lcccc}
\hline & $\log L / \mathrm{L}_{\odot}$ & $\log T_{\text {eff }}$ & $M_{\mathrm{H}, \text { env }} / \mathrm{M}_{\odot}$ & Ref. \\
\hline SN 1993J & $5.1 \pm 0.3$ & $3.63 \pm 0.05$ & $0.2-0.4$ & $(1)$ \\
SN 2008ax & $4.41-5.30$ & $3.88-4.30$ & 0.06 & $(2)$ \\
SN 2011dh & $4.90-4.99$ & $3.779 \pm 0.02$ & 0.1 & $(3)$ \\
SN 2013df & $4.94 \pm 0.06$ & $3.628 \pm 0.01$ & $0.2-0.4$ & $(4)$ \\
SN 2016gkg & $4.65-5.32$ & $3.978_{-0.158}^{+0.215}$ & - & $(5)$ \\
\hline
\end{tabular}

(1)Aldering et al. (1994); Maund et al. (2004), (2)Folatelli et al. (2015), (3)Maund et al. (2011); Van Dyk et al. (2011); Bersten et al. (2012), (4)Van Dyk et al. (2014), (5)Kilpatrick et al. (2016); Tartaglia et al. (2016)
Van Dyk, S.D., Li, W., Cenko, S.B., et al. 2011, ApJ, 741, L28 Van Dyk, S.D., Zheng, W., Fox, O.D., et al. 2014, ApJ, 147, 37 Wang, L., \& Wheeler, J.C. 2008, ARA\&A, 46, 433

Woosley, S.E., Eastman, R.G., Weaver, T.A., \& Pinto, P.A. 1994, ApJ, 429, 300 Yoon, S.-C., 2015, PASA, 32, 15

Yoon, S.-C., Gräfener, G., Vink, J. S., Kozyreva, A., \& Izzard, R. G. 2012, A\&A, 544, L11

Yoon, S.-C., Woosley, S. E., \& Langer, N. 2010, ApJ, 725, 940 